POLYTECHNIC INST OF NEH YORK BROOKLYN MICROWAVE RESE-ETC F/6 17/2 APPL ICATION OF SURFACE ACOUSTIC WAVE DEVICES TO COMMUNICATION R-ETC(U) MOV 79 WANG, H SCHACHTER, F A CASSARA AFOSR-77-\$353

UNCLASSIFIEO POLY-MRI-1403-79
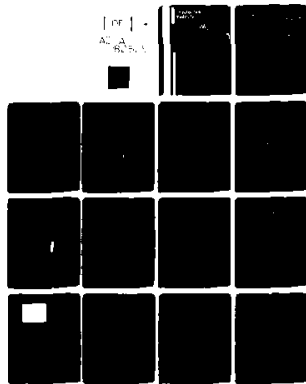
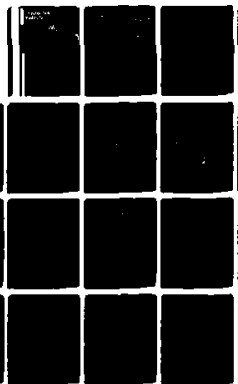

AFOSR-TR-80-0250
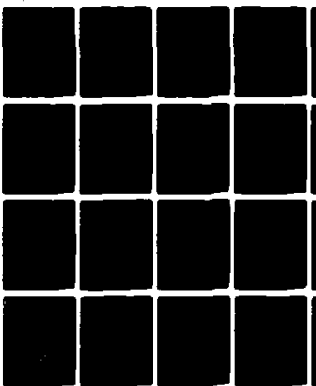
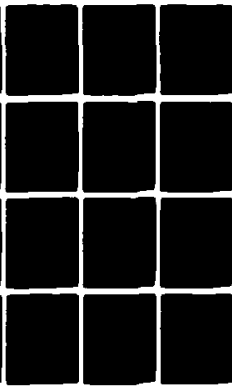

ML
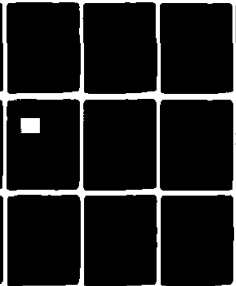

END

OATE
FILUED

$5-80$

aTc
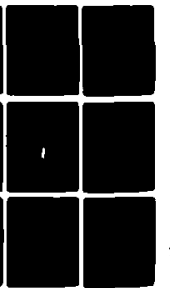


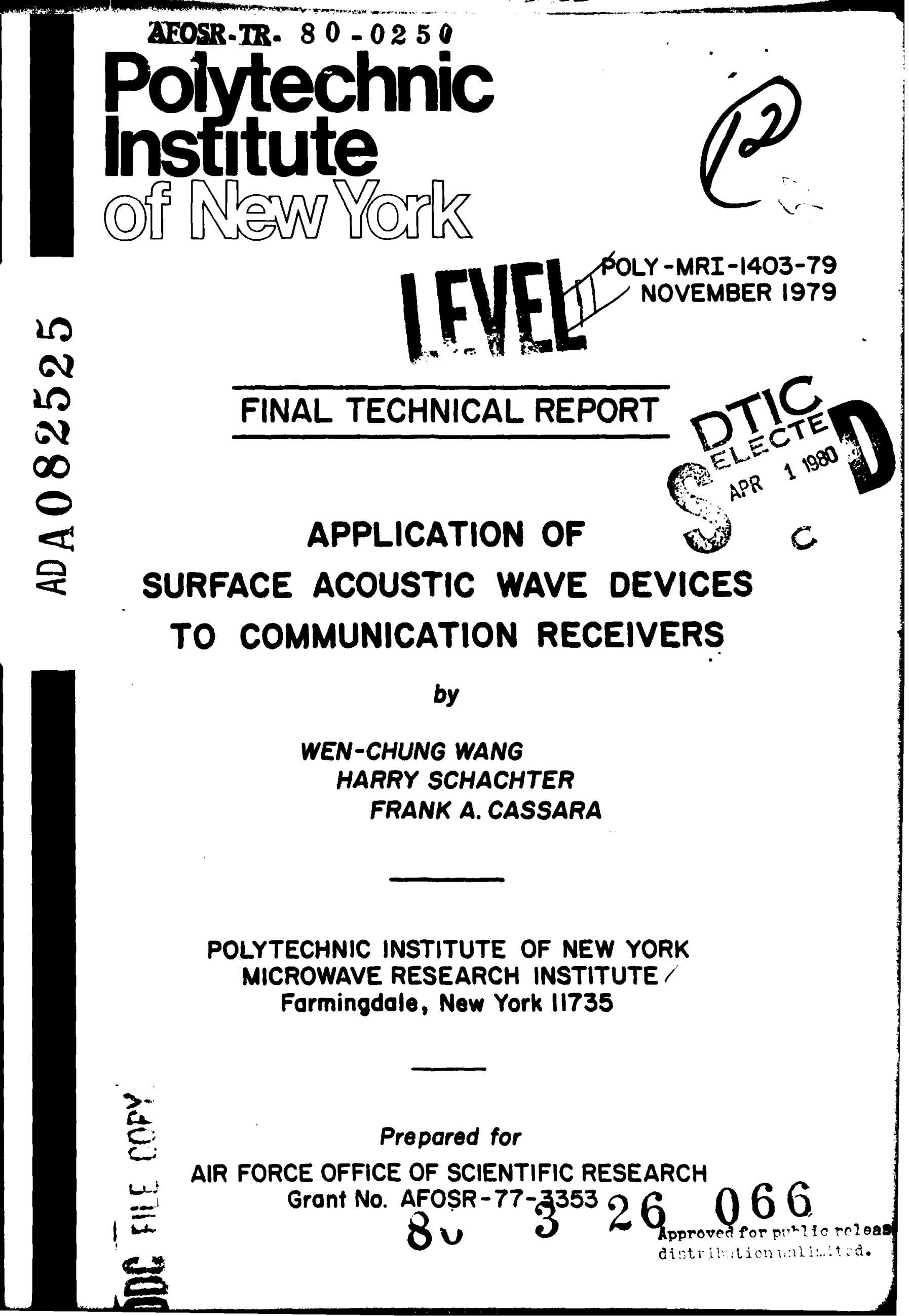


UNCLASSIFIED

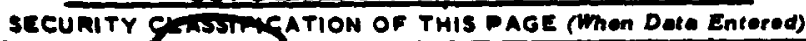

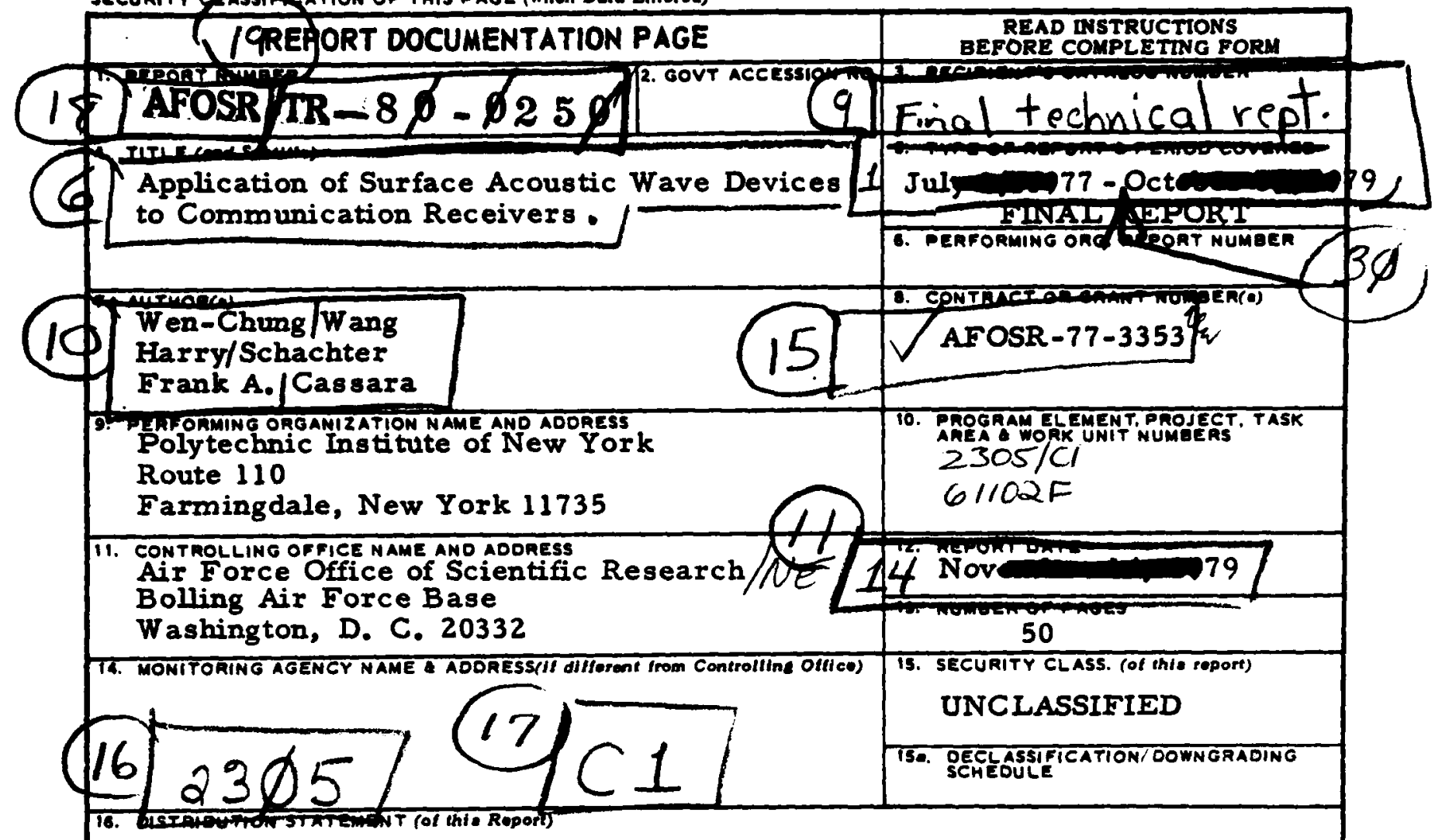

Approved for public release, distribution unlimited.

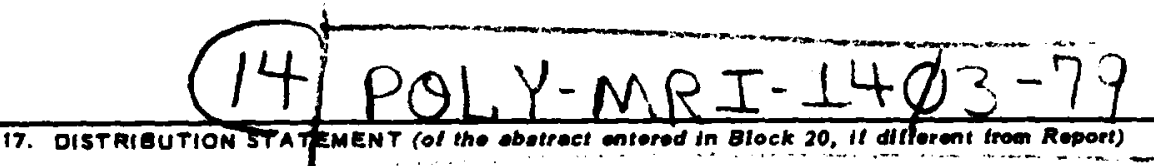

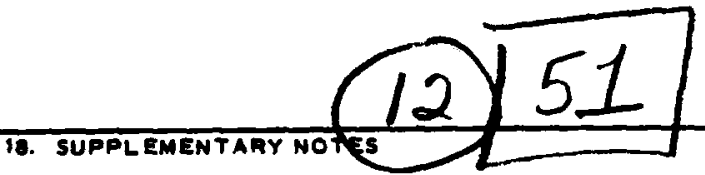

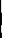

19. KEY wOROS (Continue on reverse olde il necescery and tdentlty by block number)

Surface acoustic waves, phase locked loop, voltage controlled oscillator, FM demodulator, differential phase-shift keyed demodulator, spread spectrum, thin-film technology.

20. ABsinf

This report relates to a study of acoustoelectric surface acoustic wave (SAW) devices for the detection of analog and digital communication signals. More specifically, a general SAW structure is shown to exhibit capabilities in demodulating amplitude, phase, and frequency modulated signals including those used in spread spectrum communication systems. The principle of operation for each device described is based upon acoustoelectric nonlinear interactions.

(Cont.)

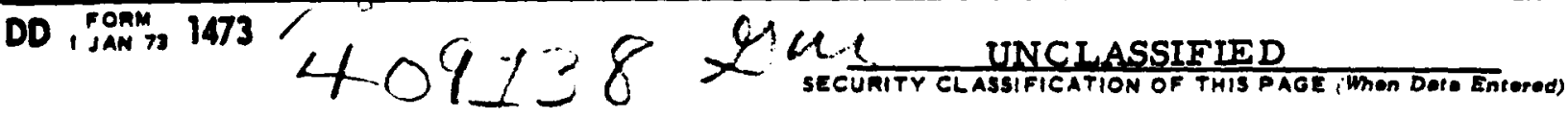


20. Abstract (Cont.)

The devices studied include the acoustoelectric phase-locked loop (PLL), SAW FM discriminator, differential phase shift-keyed (DPSK) demodulator, monolithic integrated SAW structures for the detection of apread opectrum communication signals, $\Psi$ and an acoustoelectric voltage controlled oscillator (VCO). In addition, recognizing the importance of thin-film technology in fabricating monolithic integrated structures, we initiated a thin-film technology program.

The capabilities and limitations of each of these devices were examined theoretically and experimentally. Theoretical models which provide physical insight into each demodulator's performance and design rules were advanced. Comparisons were made with known electronic strucutres. 


\section{TAB LE OF CONTENTS}

Page

ABSTRACT

I. INTRODUCTION 1

II. AN ACOUSTOELECTRIC FM DISCRIMINATOR 4

III. DIFFERENTIAL PHASE-SHIFT KEYED (DPSK) DEMODULATORS

IV. SPREAD SPECTRUM SIGNAL DETECTION 18

V. AN ACOUSTOELECTRIC PHASE-LOCKED LOOP 23

A. Principle Operation of a PLL 23

B. Acoustoelectric Phase Comparator and SAW PLL
Defining Equations

C. Stability Analysis 26

D. Bandwidth of PLL 30

E. Noise Response 31

F. Additional Results $\quad 36$

VI. ACOUSTOELECTRIC SAW OSCILLATOR AND VOLTAGE CONTROLIED OSCILLATOR 38

VII. THIN-FILM TECHNOLOGY 44

REFERENCES $\quad 46$

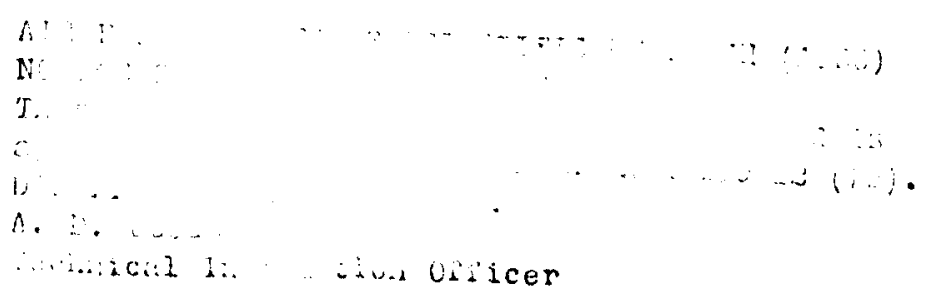




\section{ABSTRACT}

This report relates to a study of acoustoelectric surface acoustic wave (SAW) devices for the detection of analog and digital communication signals. More specifically, a general SAW structure is shown to exhibit capabilities in demodulating amplitude, phase, and frequency modulated signals including those used in spread spectrum communication systems. The principle of operation for each device described is based upon acoustoelectric nonlinear interactions.

The devices studied include the acoustoelectric phase-locked loop (PLL), SAW FM discriminator, differential phase shift-keyed (DPSK) demodulator, monolithic integrated SAW structures for the detection of spread spectrum communication signals, and an acoustoelectric voltage controlled oscillator (VCO). In addition, recognizing the importance of thin-film technology in fabricating monolithic integrated structures, we initiated a thin-film technology program.

The capabilities and limitations of each of these devices were examined theoretically and experimentally. Theoretical models which provide physical insight into each demodulator's performance and design rules were advanced. Comparisons were made with known electronic structures.

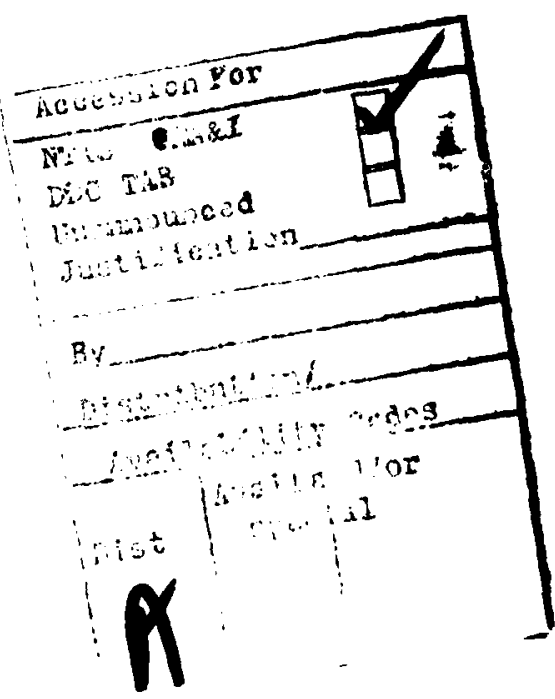




\section{INTRODUCTION}

In this work, we studied the application of acoustoelectric (AE) surface wave components to communication receivers. This new class of acoustoelectric SAW devices has exhibited capabilities in demodulating amplitude, phase and frequency modulated analog and digital communication signals. Among the devices presented are the DPSK demodulator, an FM discriminator, a PLI, and two monolithic integrated SAW structures for the detection of spread spectrum signals. The knowledge acquired during the study of the acoustoelectric PLL led to the conception of the novel SAW FM discriminator and DPSK demodulator structures. All the acoustoelectric demodulators basically can be considered as derivatives of an AE phase comparator. These devices are structurely'simple, small in size, and have been tested up to a carrier frequency of $200 \mathrm{MHz}$. Since the basic function of a communication receiver is to detect both the amplitude and phase of an incoming signal, either in analog or in digital form, we believe that this new class of AE devices can be designed to perform any signal detection efficiently at a carrier frequency limited only by IDT transducers.

The basic structure for each device is similar consisting of a piezoelectric substrate upon which a thin semiconductor plate is placed. The collinear SAW (in all cases) are excited propagating in the same direction. When the two waves propagate under the semiconductor, the piezoelectric field associated with the waves will induce space charge waves inside the semiconductor. Through the nonlinear interaction, among the piezoelectric field waves and the induced space charge waves, bigher order signals are generated and distributed along the semiconductor. However, only the higher order signal which is spatially uniform (or close to it), will have significant amplitude appearing at the output terminals across the semiconductor. This filtering action is automatically provided while the output signal is obtained by integrating along the semiconductor. This output signal is of prime concern in all the following demodulation schemes.

(1) In the case of an FM discriminator, the two collinear surface waves are of the same form and excited by the same FM input signal, how ever, they are time displaced from each other by an amount of $t_{0}$ provided by two spatially displaced input transducers. The output signal appearing across the semiconductor represents the demodulated FM signal. The requirement for successful demodulation is $t_{0} \leq \frac{1}{\Delta \omega}$, where $\Delta u$ denotes the peak 
frequency deviation of the FM signal. In general, for high frequency operation, $t_{0}$ is much smaller than the integration time $\tau$, which is the traversing time for the SAW under the semiconductor. One unique feature associated with this new type discriminator is found to be that it can either recover the modulating signal or detect the 2nd barmonic of the FM signal by simply introducing a frequency offset in the received carrier. This special feature may be valuable in coded signal transmissions. This type of discriminator is expected to be capable of accommodating wide-band FM signals with deviations up to $20 \mathrm{MH}$ sufficient for analog FM video signals as well as bigh speed data.

(2) The structure of a DPSK demodulator is practically identical to that of the FM discriminator, except here the time displacement between the two collinear surface waves $t_{0}$, has to be equal to the bit interval n $T$ " of the DPSK signal. The bit interval $T$ is generally much larger than the integration time T. These requirements for DPSK operation are seen to be opposite to that for an FM discriminator. Fr:periments have been successfully carried out at a carrier irequency of $52 \mathrm{MFE}$, a bit interval of $13 \mu \mathrm{sec}$ and an integration time of 1 Hsec. The restrictions imposed upon the bandwidth and the bit rate of the received DPSK signal by the condition $T \ll T$ will be studied. The bit error rate performance will be investigated when the received DPSK signal is corrupted by noise introduced in the communication channel.

(3) In the case of a PLL the two collinear surface waves are excited by two different signal sources, the received frequency or phase modulated signal and the output signal of the voltage controlled oscillator (VCO) and there is no time displacement between the two surface waves. The output across the semiconductor serves as the input (error signal) to the VCO. The PLL bas been successfully operated at a carrier frequency around $200 \mathrm{MHF}$. From experiments we have Iearned that (1) the stability range of the PLL depends upon the input FM modulation bandwidth of the VCO used, and (2) the hold-in range of the PLI is greatly influenced by the delay time of the traveling waves in reaching the semiconductor. The smaller this delay the larger is the hold-in range. A theory explaining this pbenomenon is presently being developed. Understanding of those observations will undoubtedly further improve the performance of the AE PLL.

Up to this date all the SAW oscillators are hybrid, i.e., conventional -lectronic amplifiers are used. An (active) integrated delay line SAW 
oscillator using a leaky SAW wave is currently under study. The principle of operation of this leaky SAW wave oscillator is very much aimilar to that of an active bulk wave oscillator developed by White and Wang. Successful results were achieved with the bulk wave oscillator for frequencies as high as $900 \mathrm{MHz}$. The reasons which prevent the active bulk wave oscillator from being a practical device are believed to be (a) the crystal used (CdS) is light sensitive and (b) the exact mode on which the oscillator is going to operate is difficult to predict. However, we believe that such a SAW oscillator will be free from the abovementioned drawbacks. The frequency range over which the bulk wave oscillator can be electronically tuned has been measured and will be reported here. The leaky SAW oscillator possesses the capability of a frequency tuning range as much as five times larger since the electromechanical coupling constant $\mathrm{K}^{2}$ for leaky SAW is at least five times larger than that of a CdS crystal. This leaky SAW oscillator will be used to implement the VCO required in the PLL.

Our search for a better and more integrated technology bas led us to develop a program of thin film deposition. Good quality $\mathrm{ZnO}$ films of thickness over $10 \mathrm{~km}$ bave been produced. High mobility InSb film is obtained. We are applying the knowledge thus developed to the fabrication of integrated devices. 


\section{AN ACOUSTOELECTRIC FM DISCRIMINATOR}

Acoustoelectric signal processors such as the convolver and corrolator are built based on (1) strong space charge nonlinearity induced by SAWs in an adjacent semiconductor and (2) the filtering action generated by spatial integration over the length of nonlinear interaction. Utilizing these special features again, a new type of FM demodulator has been developed. This type of demodulator is structurely simple, $\mathrm{Si}$ on $\mathrm{LiNbO}_{3}$, and can be operated at a carrier frequency in excess of $100 \mathrm{MHz}$.

Fig. 1 describes the basic device geometry. A frequency modulated signal $f(t)=A \cos \left(\omega_{c} t+\Delta \omega \int^{t} g(\tau) d \tau\right)$ is applied simultaneously to the pair of interdigital transducers $T_{1}$ and $T_{2}$. The transducers are separated by a small distance $\ell_{0}$. The applied FM signal will induce two collinear surface acoustic

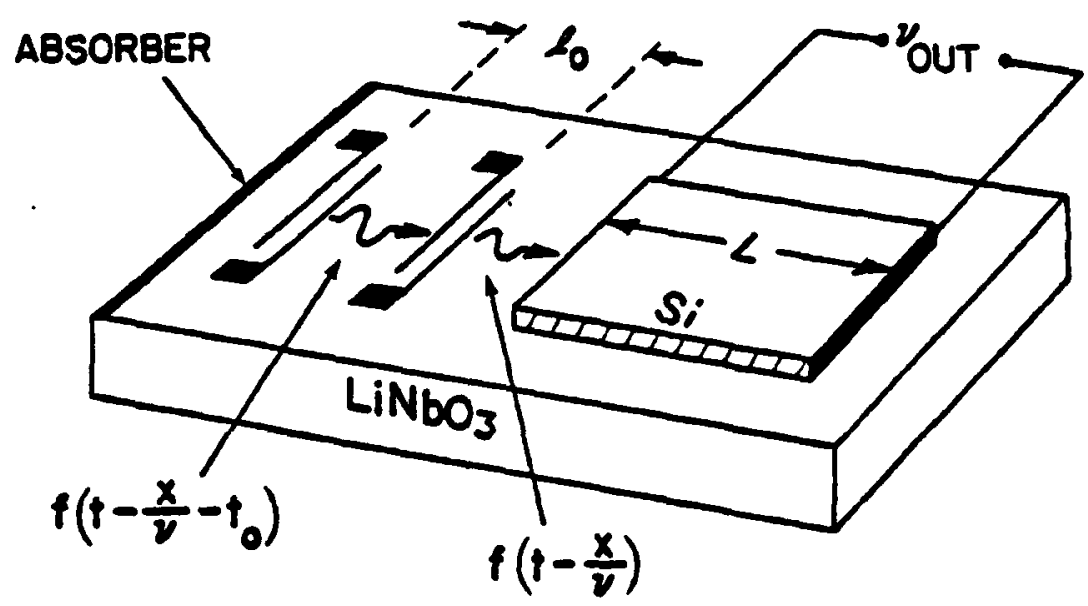

Fig. 1. Geometry of acoustoelectric FM demodulator. The delay time $L / v$ introduced by the spatial separation of the two input transducers.

waves which are propagating in the same direction and with a time separation of $t_{0}=l / v$, where $v$ is the SAW velocity. When the two waves propagate under the semiconductor, the piezoelectric field associated with the waves will induce space charge waves inside the semiconductor. Through the nonlinear interactions among the induced space charge waves and the piezoelectric field waves, 
many higher order signals are generated. However, after integrating along the semiconductor length $L$, only the second order term, which performs the FM demodulation, is of significant amplitude and $c a n$ be detected across the semiconductor terminals.

\section{Consider}

$$
e_{1}(t)=A_{1} \cos \left(w_{c} t+m(t)\right)
$$

and

$$
e_{2}(t)=A_{2} \cos \left(\omega_{c}\left(t-t_{0}\right)+m\left(t-t_{0}\right)\right)
$$

the signals applied at $T_{1}$ and $T_{2}$ with $\omega_{c}$ the carrier frequency and $m(t)$ the modulation.

The induced electric fields and charges due to the input signal will be

$$
\begin{aligned}
& E_{1}(t, y, x)=A_{1} E_{1}(y) \cos \left(w_{c}\left(t-\frac{x}{v}\right)+m\left(t-\frac{x}{v}\right)+\theta_{1}(y)\right) \\
& p_{1}(t, y, x)=A_{1} \bar{p}_{1}(y) \cos \left(w_{c}\left(t-\frac{x}{v}\right)+m\left(t-\frac{x}{v}\right)+\phi_{1}(y)\right) \\
& E_{2}(t, y, x)=A_{2} E_{2}(y) \cos \left(w_{c}\left(t-t_{0}-\frac{x}{v}\right)+m\left(t-t t_{0}-\frac{x}{v}\right)+\theta_{2}(y)\right) \\
& B_{2}(t, y, x)=A_{2} \bar{p}_{2}(y) \cos \left(w_{c}\left(t-t_{0}-\frac{x}{v}\right)+m\left(t-t_{0}-\frac{x}{v}\right)+\phi_{2}(y)\right)
\end{aligned}
$$

where the assumption was that the amplitude of the given signals varies little over the range of frequencies used, since the frequency deviation is quite small in comparison with the carrier frequency.

These charges and fields give rise to currents at sum and difference frequencies. Neglecting diffusion, the open current voltage in the $x$-direction will be

$$
V(t)=\frac{\mu}{\sigma} \int_{0}^{L} J_{N L} d x=\frac{\mu}{\sigma} \int_{0}^{L}\left(E_{1} \rho_{1}+\rho_{2} E_{2}+\rho_{1} E_{2}+\rho_{2} E_{1}\right) d x .
$$

Specifically, the low frequency voltage will be (neglecting de and higher frequency terms) 


$$
\begin{aligned}
v(t) & =\frac{\mu}{\sigma} \int_{0}^{L}\left[E_{1} \bar{\rho}_{2} \cos \left(\omega_{c} t_{0}+m\left(t-\frac{x}{v}\right)-m\left(t-t_{0}-\frac{x}{v}\right)+\theta_{1}(y)-\phi_{2}(y)\right)+\right. \\
& \left.+E_{2} \bar{p}_{1} \cos \left(\omega_{c} t o m\left(t-\frac{x}{v}\right)-m\left(t-t_{0}-\frac{x}{v}\right)+\phi_{1}(y)-\theta_{2}(y)\right)\right] d x .
\end{aligned}
$$

Under the conditions $t_{0}\left(t-\frac{x}{v}\right) \ll 1$, we get (again neglecting de terms)

$$
V(t)=\frac{\mu A_{1} A_{2} t}{2 \sigma} \int_{0}^{L} \dot{m}\left(t-\frac{x}{v}\right)\left[E_{1} \bar{p}_{2} \sin \left(\omega_{c} t t_{0}+\theta_{1}-\phi_{2}\right)+E_{2} \bar{p}_{1} \sin \left(\omega_{c} t_{0}+\phi_{1}-\theta_{2}\right)\right] d x
$$

i.e.,

$$
V(t)=B(y) \int_{t-\frac{L}{V}}^{t} \dot{m}(\lambda) d \lambda
$$

where $L$ is the total integration length in the $x$ direction. Finally, if $\dot{m}(x)$ changes slowly in the interval $\frac{\mathrm{L}}{\mathrm{v}}$, then

$$
V(t)=B(y) \frac{I}{v} \dot{m}(t)
$$

Experiments confirming these results have been reported and shown in the oscillograms of Fig. 2. The top traces designate the shapes of the input modulation signal and the lower traces are the corresponding demodulated signal. Successful FM demodulation is clearly achieved.

An exact theoretical analysis for a particular arbitrary input modulation signal such as a triangle is quite cumbersome. The mixing among the fourier components of the input signal is expected to be the main source of distortion. In order to understand the performance of this new type of demodulator in relative detail, we examine a simple case in which $m(t)=\beta \sin \omega_{m} t$, where $\beta$ is the modulation index and is equal to $\Delta w / \omega, \Delta w$ is the peak frequency deviation, and $\omega_{\mathrm{m}}$ the modulation rate. For this simple case a close form representation for the output voltage across the semiconductor is obtained and expressed in the following: 


$$
\begin{aligned}
& v_{\text {o. } c_{0}}=\int_{0 .}^{L} K_{2} 2 f\left(t-\frac{x}{v}\right) f\left(t-\frac{x}{v}-t_{0}\right) d x
\end{aligned}
$$

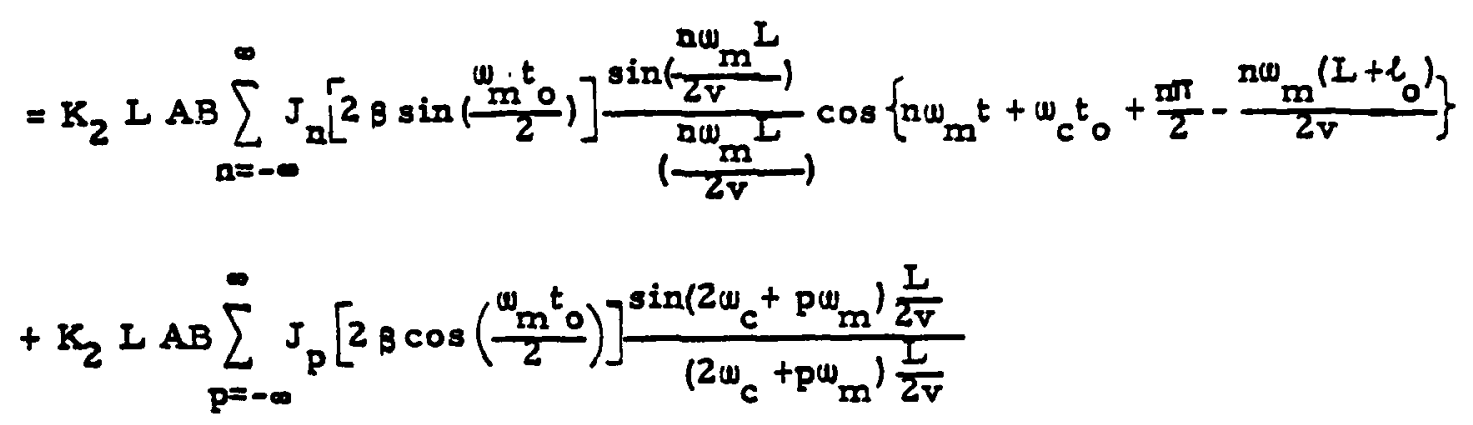

$$
\begin{aligned}
& \cos \left\{\left(2 w_{c}+p w_{m}\right) t+w_{c} t_{0}-\frac{w_{c} L}{v}-\frac{p w_{m}\left(I+l_{0}\right)}{2 v}\right\}
\end{aligned}
$$

where $J_{n}\left(2 g \sin \frac{D_{m}^{t} o}{2}\right)$ is the nth order Bessel function.

We note for the special case $\omega_{m} t_{0}<<1$, the argument of the Bessel function becomes $\Delta \omega t_{0}$.

In Eq. (4) $\mathrm{K}_{2}$ is a constant in terms of the 2nd order nonlinear coefficient and other device parameters such as carrier density, mobility, etc.

Eq. (4) reveals that the novel SAW device does indeed demodulate the received FM signal ( $n= \pm 1$ terms) provided $\omega_{c} t_{0} \neq n \pi$. It is noted in the above expression that the action of spatial filtering comes in the form of $\frac{\sin \theta}{\gamma}$. As a numerical example, let us consider the case when $\frac{L}{v} \approx 3 \mu \mathrm{sec}$, $\omega_{\mathrm{m}} \approx 8 \pi \times 10^{3} \mathrm{rad} . / \mathrm{sec}$. and $\omega_{c} \approx 2 \pi \times 10^{8} \mathrm{rad} . / \mathrm{sec}$. The $\frac{\sin \theta_{n}}{\theta_{n}}$ factor $\left(\theta_{n}=n w_{m} L / 2 v\right.$, associated with $\left.J_{n}\right)$ approaches to 1 for $n \leq 2$, and the factor $\sin \theta_{p} / \theta_{p}$ (associated with $J_{p}$ ) approaches to zero for any $p$. Therefore, the only term remaining in the open circuit voltage expression is that related with the modulation signal. We also note that in order to maintain linearity, i. e., discriminator output voltage to be linearly related to input frequency excursions 
$(\Delta \omega)$, we require $\Delta w t_{0}<1$. For example, if a frequency deviation, $\Delta f=20 \mathrm{MHz}$ is needed, one requires a delay of $t_{0} \leq 5 \mathrm{n}$ sec. and a carrier frequency $f_{c} \approx 10^{3} \mathrm{MHz}$. A uaique feature inherent in this device is that one can adjust $\omega_{c} t_{0}$ to select a set of desired order, (even, odd or both) of Bessel functions to operate with. Experimental results are in agreement with the derived expression.

A typical set of experiments are described here in which $t_{0}$ is set at $0.34 \mathrm{\mu sec}$. The oscillograms in Fig. 2 are taken at constant carrier frequency, $95 \mathrm{MHz}$ at varying frequency deviations. (At this carrier frequency only odd harmonics are observed.) The observed disappearances (first zeros) of fundamental, third harmonic and fifth harmonic are corresponding to $\Delta u t_{0}=3.74,6.4$ and 8.8 respectively. They are compared with theoretical predictions of $\Delta u t_{0}=3.81,6.38$ and 8.77. The oscillograms in Fig.I3 are taken under conditions of constant frequency deviation, but varying carrier frequencies. In oscillogram(3a) only the odd (fundamentai) is predominant, in (3b) both even and odd are present, in(3c) only the even (second) is predominant. From (3a) to(3c) the change in weto is $\left\{\left(w_{c} t_{0}\right)_{a}-\left(w_{c} t_{0}\right)_{c}\right\}=0.54 \pi$, compared with theoretical prediction $0.5 \pi$. Fig. 4 gives the comparison between the shapes of the input modulation signal $g(T)$ and that of the device's output demodulated signal. Clearly successful FM demodulation is achieved even for the case of nonsinusoidal modulation. The major contribution to the theoretical output distortion is due to the following 4th order nonlinear term

$$
\begin{aligned}
& \int_{0}^{L} K_{4} f^{2}\left(t-\frac{x}{v}\right) f^{2}\left(t-\frac{x}{v}-t_{0}\right) d x \\
& =C_{d . c_{0}}+K_{4}\left(\frac{A^{2} B^{2}}{4}\right) L \sum_{n=-\infty}^{\infty} J_{n}\left(2 \Delta \omega t_{0}\right) \cos \left\{n \omega_{m} t+2 \omega_{c} t_{0}+\varphi\right\} \\
& -8-
\end{aligned}
$$


where $\mathrm{K}_{4}$ is related with the 4 th order nonlinear coefficient and $\mathrm{K}_{4} \ll \mathrm{K}_{2}$. Comparing eq.(I3) with Eq. (4) one sees that if one desires odd harmonics only by adjusting $\left\{w_{c} t_{0}+\varphi\right\}=\frac{\pi}{2}$, then, the 4th order nonlinear term of Eq. (5) will predominantly contain even harmonic terms which provide distortion. 


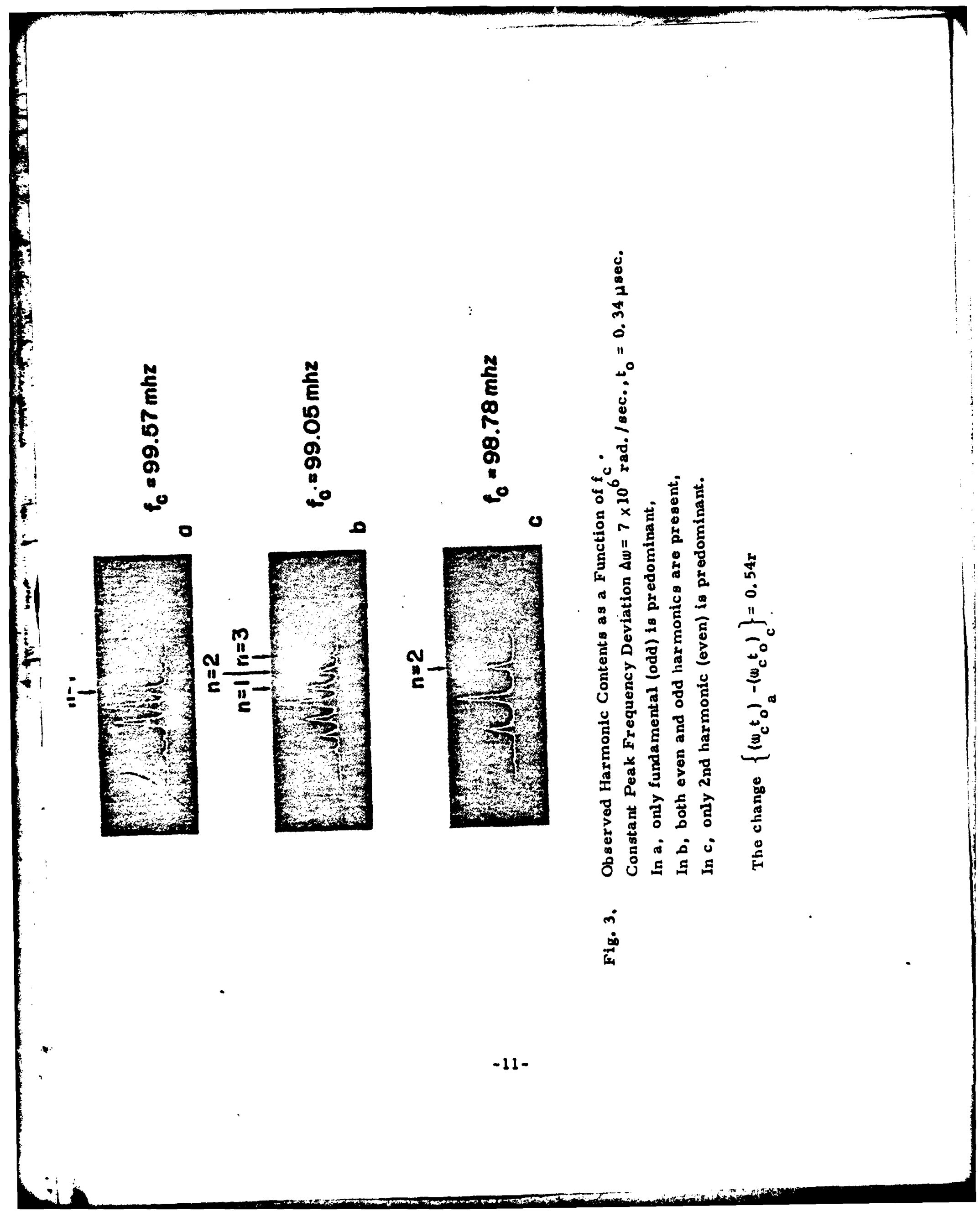




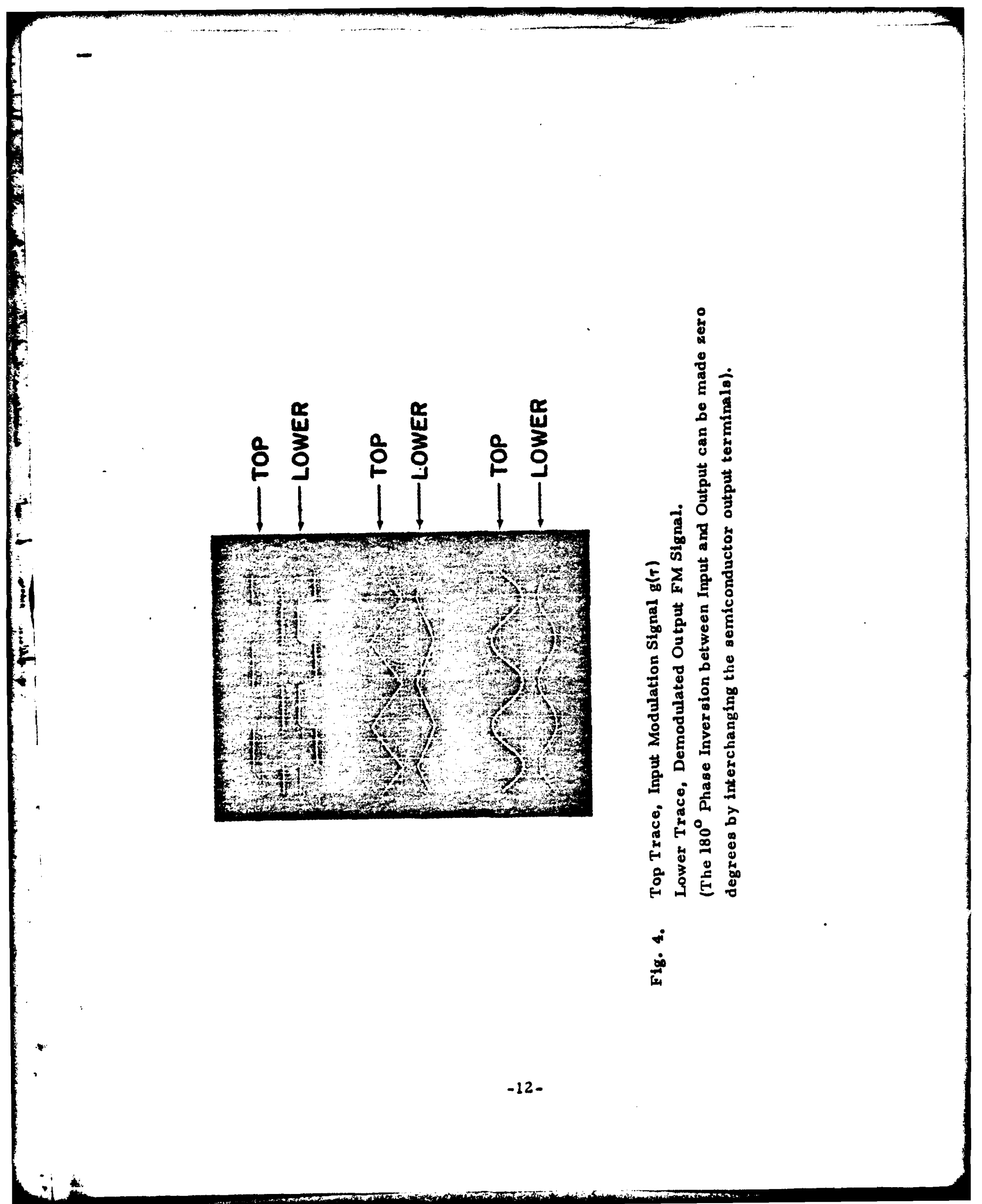


III. DIFFER ENTIAL PHASE-SHIFT KEYED (DPSK) DEMODULATORS

Phase shift keying (PSK) is a well-known technique ${ }^{2}$ for phase modulating carrier signals with digital communication signals. Demodulation of PSK is accomplished by synchronous detection. Unfortunately, generation of the reference signal required for the synhcronous detector is relatively difficult to obtain. Differential phase shift keying (DPSK) is an alternate technique of transmitting a PSK type signal that enables the receiver's synchronous reference signal to be derived very simply directly from the received signal. A disadvantage of DPSK is that bit errors tend to occur in pairs and, therefore, bit error rate performance is not as good as synchronous PSK. However, the advantage of hardware simplicity has made DPSK a popular modulation technique. A block diagram of the DPSK transmitter is shown in Figure 1.

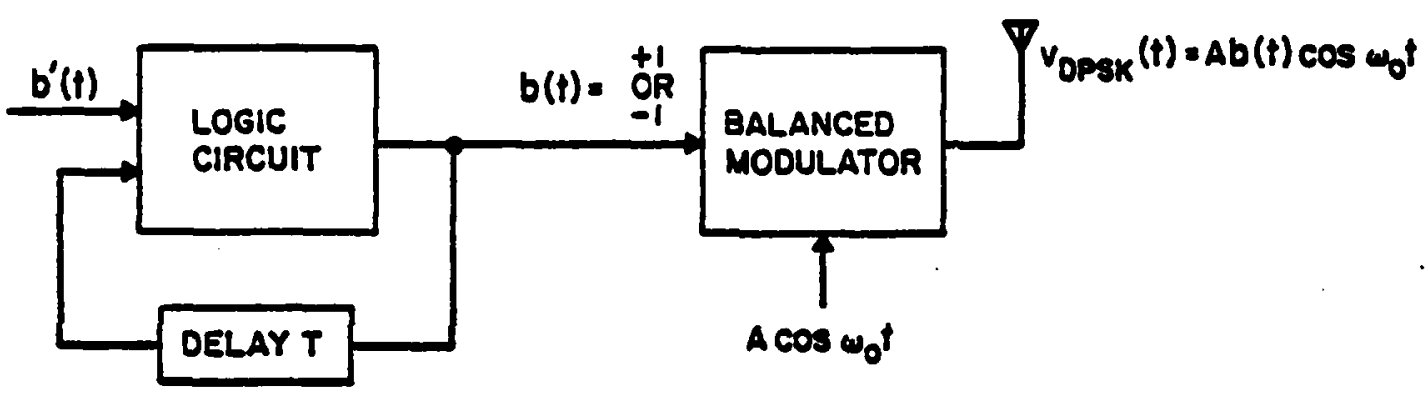

Fig. 1. DPSK modulator.

In this figure, $b^{\prime}(t)$ describes the binary digital data to be transmitted, $i . e .$, the message signal. $b(t)$ denotes another binary sequence obtained from $b^{\prime}(t)$ using the following rule:

$$
b(t)=b(t-T) b^{\prime}(t)
$$

where $T$ corresponds to one bit interval. Thus, whenever $b^{\prime}(t)$ is a logic 1 in some bit interval, $b(t)$ in that interval does not change from its value in the preceding interval. When $b^{\prime}(t)$ is a logic $0, b(t)$ does change. Next, $b(t)$ modulates a carrier $A \cos w_{0} t$ such that whenever $b(t)$ is a logic 1 the transmitted signal $V_{D S P K}=A \cos w_{0} t$ and whenerer $b(t)$ is a logic $0, V_{D P S K}=-A \cos w_{0} t$.

The block diagram of the conventional DPSK demodulator is shown in Fig. 2. 


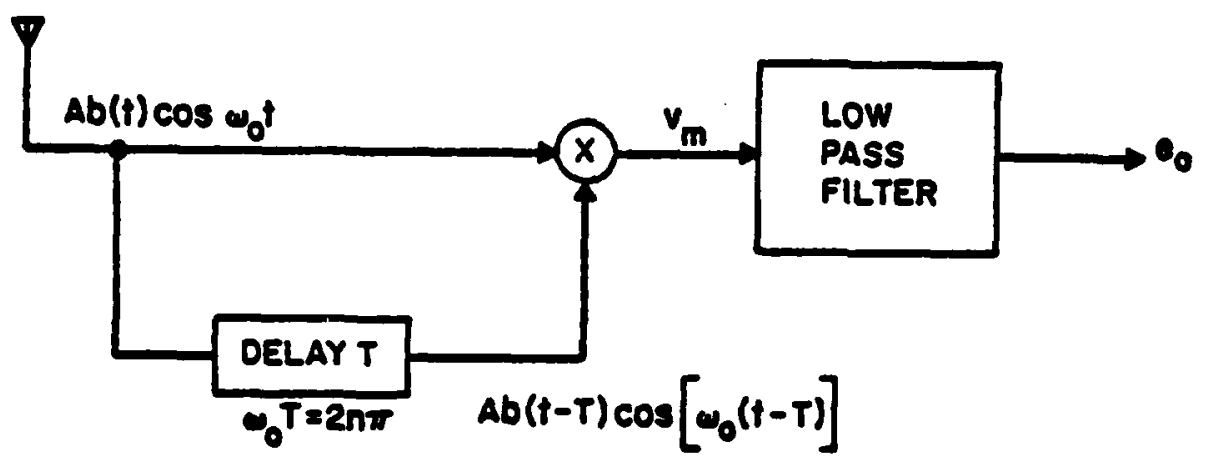

Fig. 2. Conventional DPSK demodulator.

The multiplier outpat, $v_{m}$, can readily be shown to be:

$$
v_{m}(t)=\frac{A^{2} b(t) b(t-T)}{2} \cos w_{0} T+\text { higher frequency terms }
$$

By designing $w_{0} T=2 \pi n$ where $n=$ integer, we obtain

$$
e_{0}(t)=\frac{A^{2}}{2} b(t) b(t-T)
$$

By multiplying both sides of Eq. (1) by $b(t-T)$, we recognize $e_{0}(t)=\left(A^{2} / 2\right) b^{\prime}(t)$ and hence successful demodulation of the original data stream $b^{\prime}(t)$ is achieved. ACOUSTOELECTRIC DPSK DEMODULATOR

An acoustoelectric DPSK demodulator was conceived and its structure is illustrated in Figure 3.

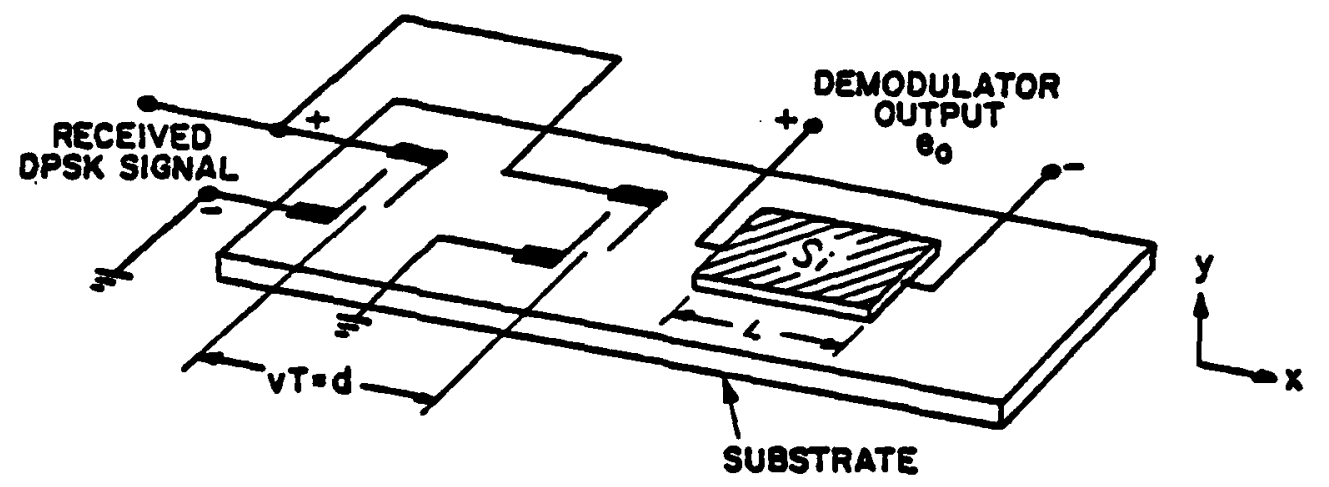

Fig. 3. Acoustoelectric DPSK demodulator.

In this structure the received signal is simultaneously applied to two interdigital SAW transducers displaced by $d=v T$ where $v$ denotes the velocity of the acoustic Rayleigh surface wave in the piezoelectric substrate and $I$ again 
denotes the bit interval. When the sum of the two surface acoustic waves propagate under the semiconductor (see Fig. 3), the piezoelectric field associated with the waves will induce space charges and electric fields inside the semiconductor. Through the nonlinear interactions among the induced space charges and induced electric fields, numerous higher order signals are generated. After integrating along the silicon semiconductor length $L$, only the term which performs the DPSK demodulation is significant and can be detected acrose the semiconductor terminals as shown in Fig. 3. Quantitatively, the electric field in the silicon can be expressed as:

$$
\begin{aligned}
E(t, x, y)= & E_{0}(y, w)\left\{b\left(t-\frac{x}{v}\right) \cos \left[\omega_{0}\left(t-\frac{x}{v}\right)+\theta_{E}(y, w)\right]\right. \\
& \left.\cdots+b\left(t-T-\frac{x}{v}\right) \cos \left[\omega_{0}\left(t-\frac{x}{v}-T\right)+\theta_{E}(w, y)\right]\right\}
\end{aligned}
$$

The induced charge density in the silicon plate is given by

$$
\alpha t, x, y)=\rho_{0}(y, \omega)\left\{b\left(t-\frac{x}{v}\right) \cos \left[\omega_{0}\left(t-\frac{x}{v}\right)+\theta_{\rho}(y, \omega)\right]+b\left(t-T-\frac{x}{v}\right) \cos \left[\omega_{0}\left(t-\frac{x}{v}-T\right)+\theta_{\rho}(y, \omega)\right]\right\}
$$

where $E_{0}(y, w)$ and $p_{0}(y, w)$ are assumed to vary slowly with $w$ in the vicinity of the carrier frequency $w_{0}$. The open-circuit voltage collected across the silicon plate is approximated by

$$
e_{0}=\frac{\mu}{0} \int_{0}^{L} E(t, x, y, w) \rho(t, x, y, w) d x
$$

substitution of Eqs. (4) and (5) into Eq. (6) yields:

$$
e_{0} \approx K \int_{0}^{L} b\left(t-\frac{x}{v}\right) b\left(t-T-\frac{x}{v}\right) \cos w_{0} I d x+e_{D C}+\text { high frequency terms }
$$

where $\mathrm{K}$ denotes a constant and ${ }^{\mathrm{E}} \mathrm{DC}$ denotes the average component appearing across the silicon. Neglecting the higher frequency terms (which are filtered out by the integration) and making the transformation of variables $u=t-x / v$, we obtain

$$
e_{0} \approx K \int_{t=\frac{L}{v}}^{t} b(u) b(u-I) d u+e_{D C}
$$


If we make the restriction $\frac{L}{v} \ll T$, the integrand is a constant over the limits of integration and we obtain,

$$
e_{0} \approx K^{\prime} b(t) b(t-T)=K^{\prime} b^{\prime}(t)
$$

revealing that the device can theoretically successfully demodulate the received DPSK signal.

The device structure shown in Fig. (3) was constructed using a lithium niobate piezoelectric substrate, with $52 \mathrm{MHz}$ aluminum transducers. The delay between interdigital transducers was $13 \mu \mathrm{sec}$. The resistivity of the silicon plate was $70 \mathrm{ohm}-\mathrm{cm}$ with length $L=0.1 \mathrm{~cm}$. The velocity of the acoustic wave in the substrate is $v=3 \times 10^{5} \mathrm{~cm} / \mathrm{sec}$. Hence, $\frac{L}{v}=1 \mu \mathrm{sec} \ll<\mathrm{T}$ and the restriction following Eq. (8) is satisfied. The received DPSK signal A b $(t)$ cos $\omega_{0} t$ consisted of an alternating sequence of ones and zeros. The binary sequence $b(t)$ is shown in Fig. 4. From Eq. (1), we recognize that the data sequence $b^{\prime}(t)$ containing the message signal can be obtained by multiplying $b(t)$ by $b(t-T)$. This is also shown in Figure 4.

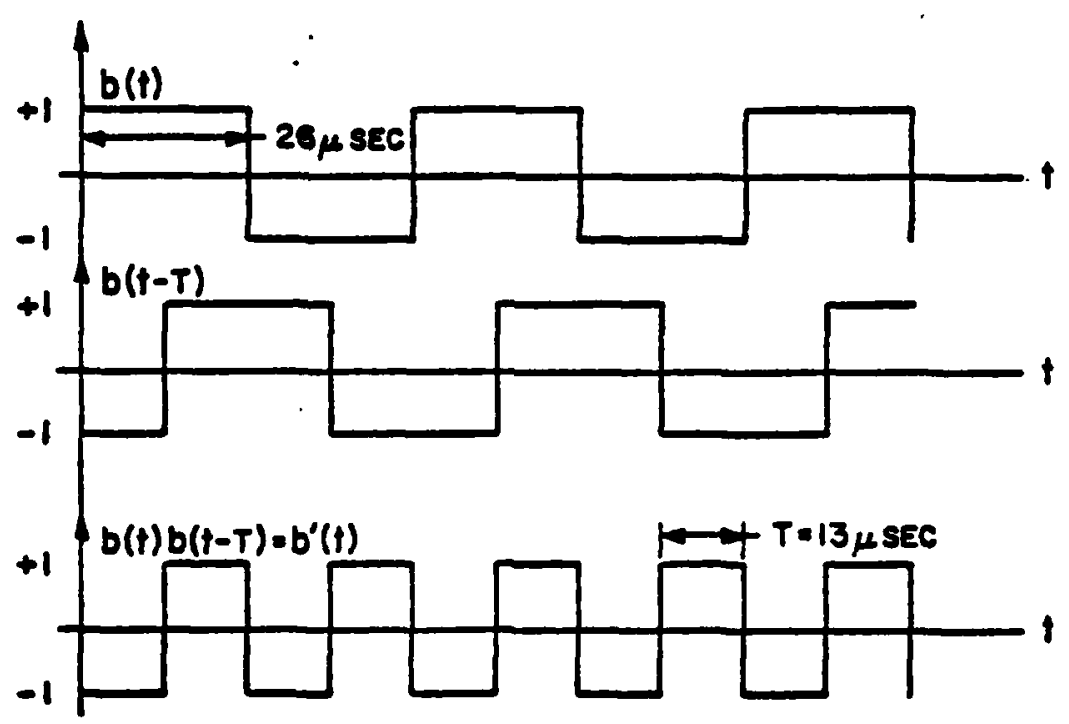

Fig. 4. DPSK received data streams $b(t), b(t-T)$, and $b^{\prime}(t)=b(t) \cdot b(t-T)$. 
Figure 5 describes an oscillogram of the binary signal $b(t)$ (top trace) which was used to phase shift modulate the received RF carrier and the acoustoelectric DPSK demodulator output binary sequence $b^{\prime}(t)$. These results reveal successful demodulation of the received signal.

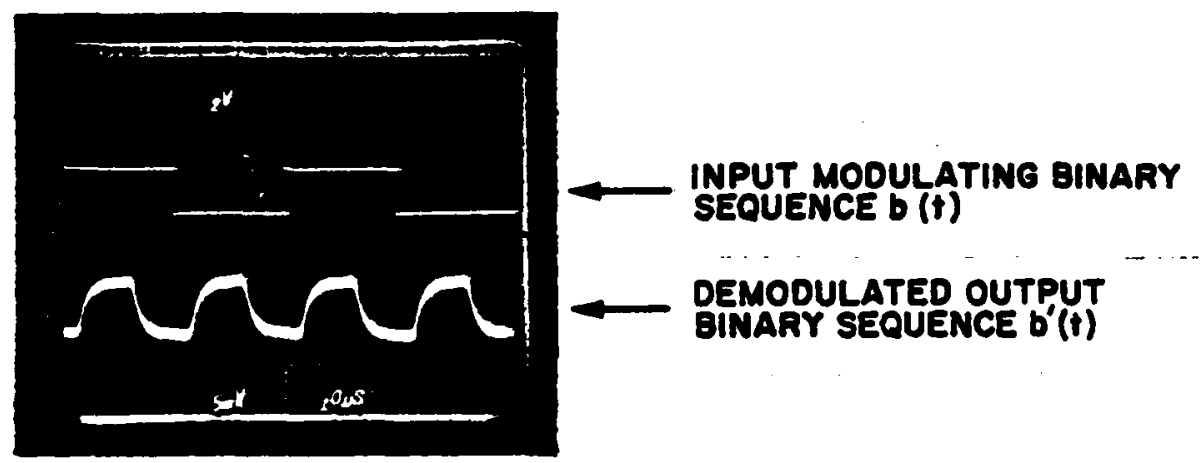

Fig. 5. Acoustoelectric DPSK demodulator responses

$$
\left(T=13 \mu \mathrm{sec}, \frac{L}{V}=1 \mu \sec \ll T, f_{0}=\frac{\omega_{0}}{2 \pi}=52.422 \mathrm{MHz}\right)
$$

\section{FUTURE GOALS}

.Our future research objectives include the theoretical and experimental investigation of the novel SAW DPSK demodulator to determine its capability and limitations. Experimental devices will be fabricated utilizing our new thin-film technology. Restrictions imposed upon bandwidth of the received DPSK signal and the bit rate by the interaction time, $T$, will be determined. Distortions of pulse signal $b(t)$ due to the fact that $E_{0}(y, w)$ and $n_{0}(y, w)$ in Si vary with frequency will be analyzed deriving thus a lower limit for $T$. Practical rules of design will be advanced. Carrier frequencies as bigh as $200 \mathrm{MH}$ will be used in the experimental study. Bit error rate performance will be determined when the received DPSK signal is corrupted by white gaussian noise introduced in the communication channel. Comparisons will be made on the conventional demodulators. 


\section{SPREAD SPECTRUM SIGNAL DETECTION}

We extended this study to the case when the DPSK is utilized for the base band data modulation in a direct sequence spread spectrum communication signal. Two structures were conceived to detect such signals.

The first structure is shown in Figs. 1 and 2. In Fig. I we present the block diagram of a real-time correlator ${ }^{7}$ for demodulation of spread spectrum signals. The inputs to the correlator consist of the received signal and is compared to the locally generated referenced signal. The correlator output is processed by the previously described DPSK demodulator to recover the DPSK data. Fig. 2 represents a physical realization using $A E$ devices. The correlator is composed of a semiconductor plate sandwiched between two piezoelectric plates; one made of $\mathrm{LiNbO}_{3}$ and the other BGO. The incoming signal is applied to the interdigital transducer on the BGO (input 1) and the locally generated reference signal is applied to the transducer on the $\mathrm{LiNbO}_{3}$ (input 2). Since the acoustic velocity of the generated Rayleigh waves in the two piezoelectric plates are different, in order to match them spatially the carrier frequency of the applied correlator inputs must be different. The output of the correlator is taken at terminals 3 and 4 across the multilayer structure and simultaneously applied to two interdigital transducers on the aurface of the $\mathrm{LiNb}_{3}$ displaced one data bit delay apart. Finally, the opencircuit voltage taken along the semiconductor plate at terminal 5 will represent the detected data bits. Notice that since both the received signal and the reference signal travel in the same direction, no time reversal of the pseudo-random (pa) code is necessary. Another important feature of this device is its compactness.

The second structure is presented in Figs. 3 and 4. Fig. 3 describes a block diagram of the demodulator in which the comparison between the received spread spectrum signal and the locally generated reference signal is accomplished with a convolver. As in the previous structure, the convolver output is processed by the DPSK demodulator presented in this work. Fig. 4 presents the physical realization of the tevice. The received signal and the reference signal are applied such that the generated Rayleigh waves travel in opposite directions beneath a semiconductor plate. The voltage developed across the layered structure is simultaneounly applied to two interdigital traneducers displaced one data bit interval apart. The outpat at terminal 5 again yields the demodulated data bits. Our future research objectiven include fabricating such structures utilizing our recently developed $\mathrm{ZnO}$ thin-film technology and experimentally and analytically evaluating their performance capabilities. Current technology indicates that BI product factors as bigh as several thousand are feasible. 


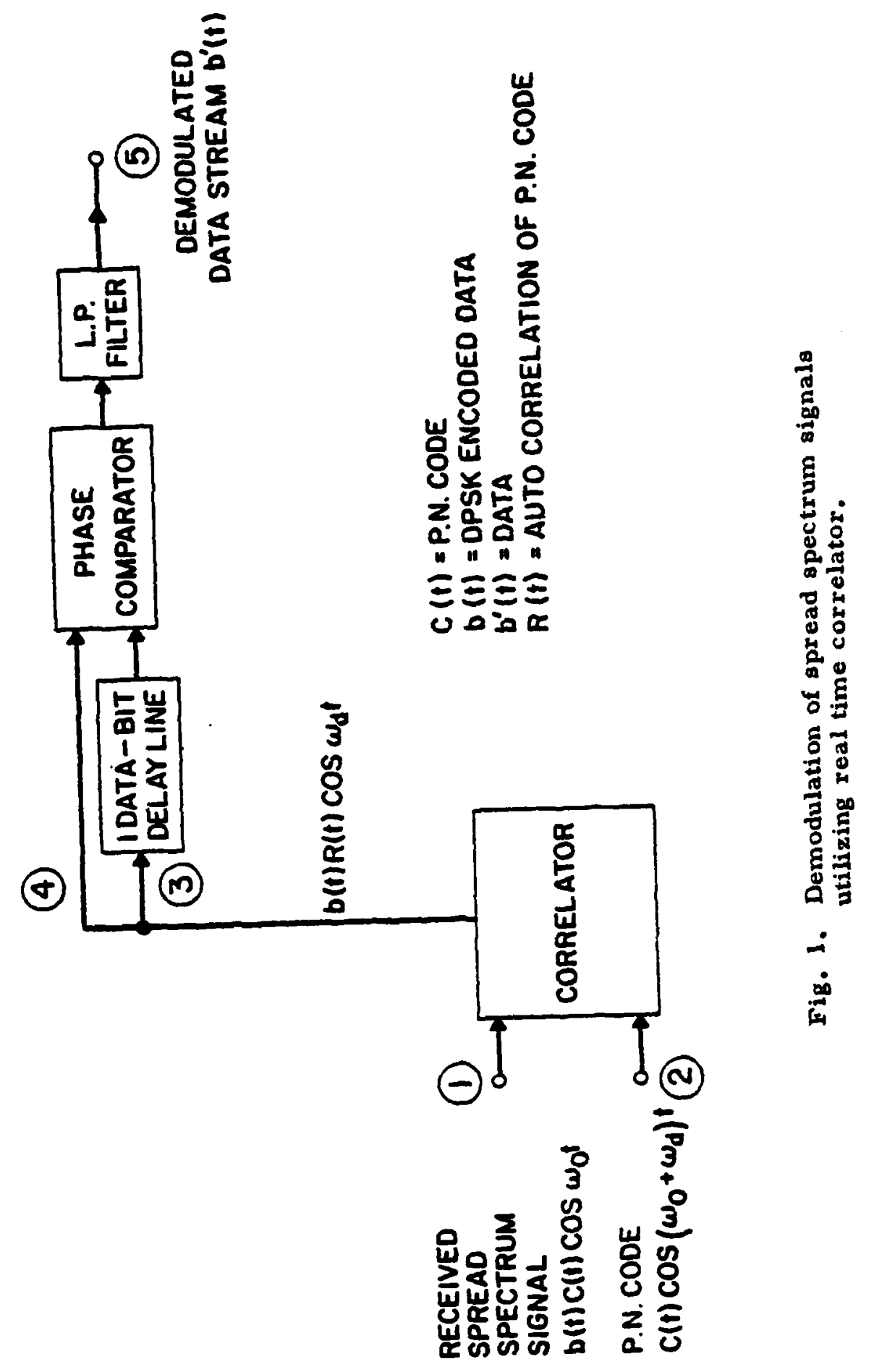



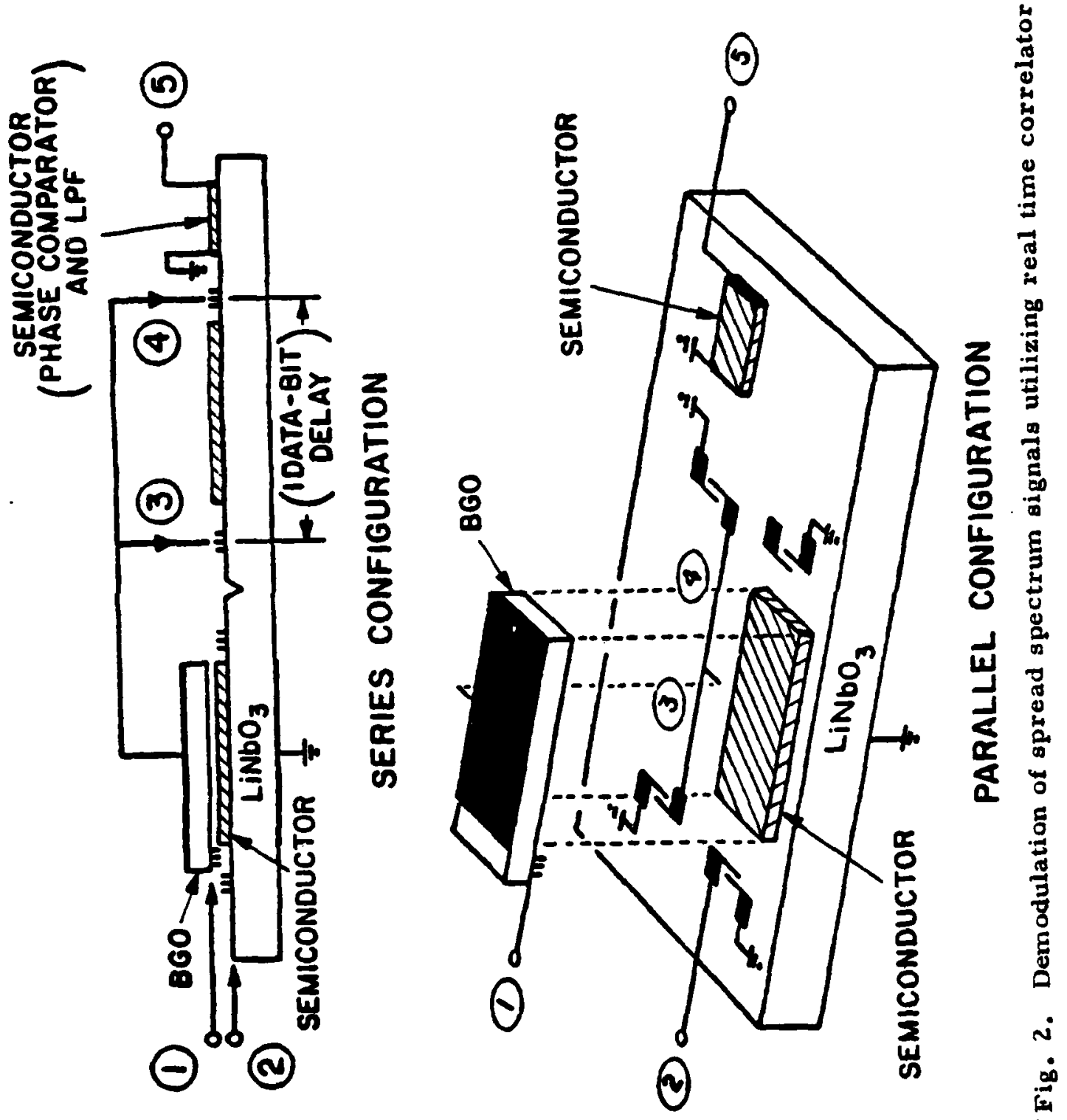


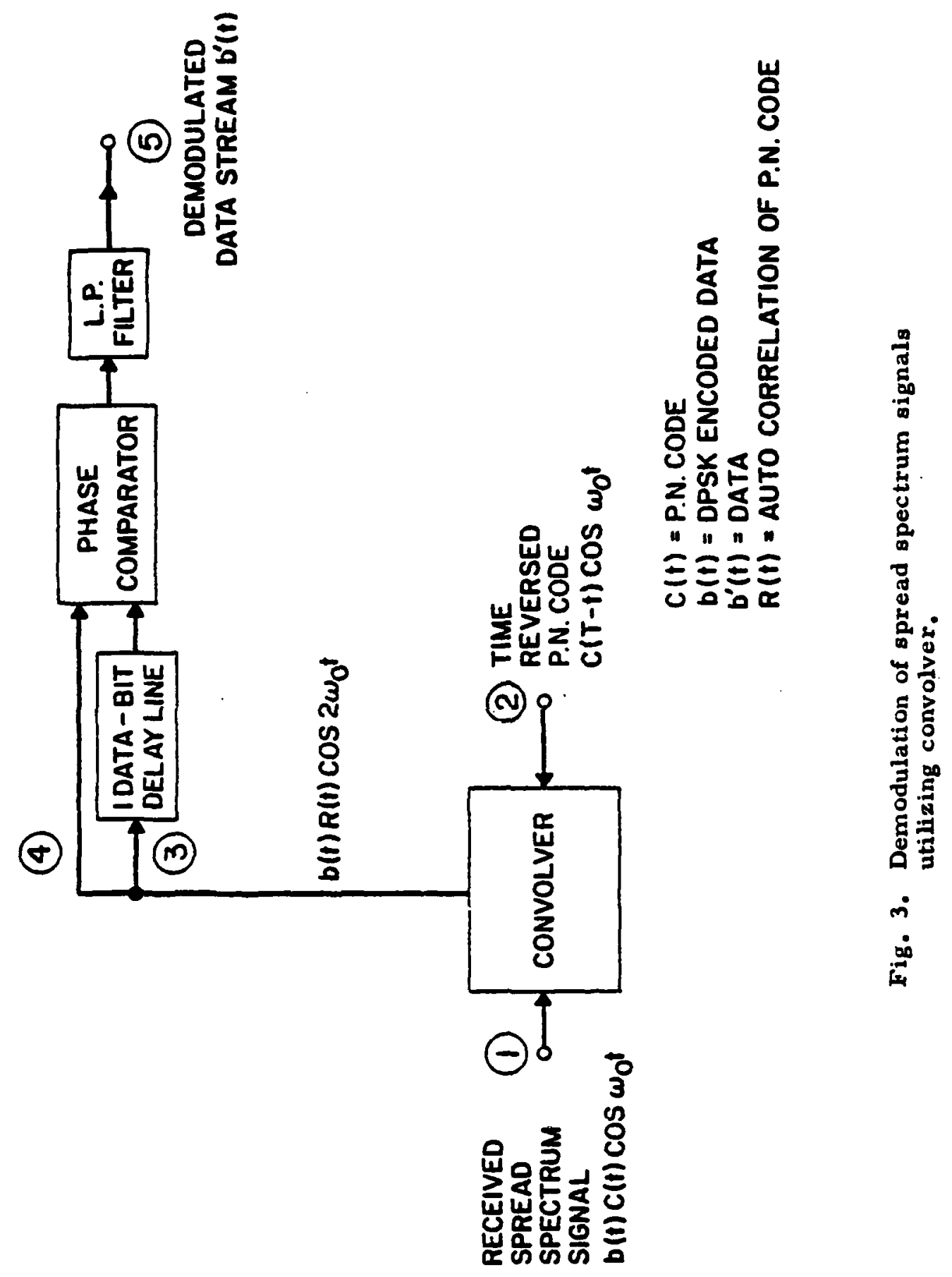



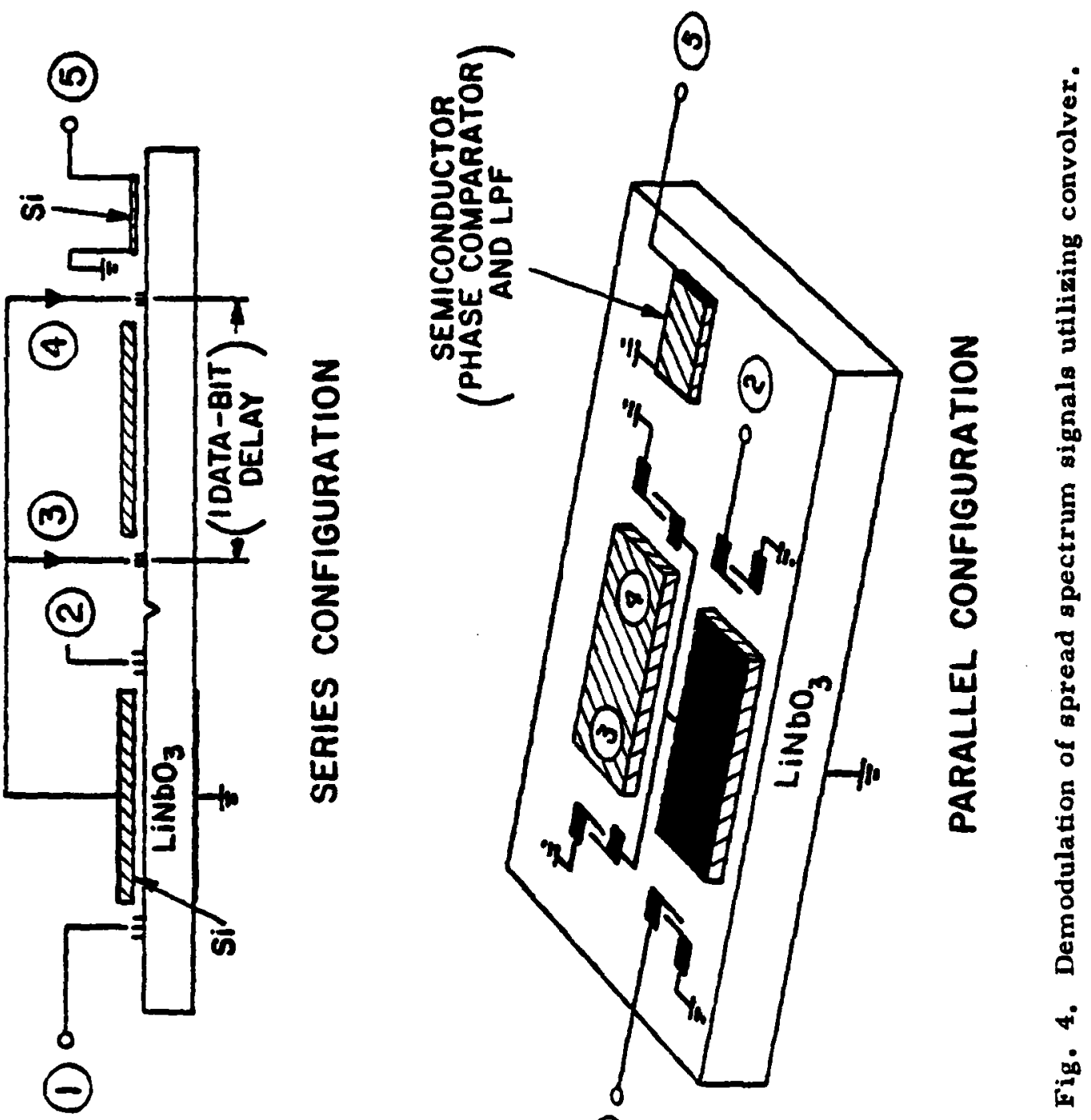

$\theta$ 


\section{AN ACOUSTOELECTRIC PHAASE-LOCKED LOOP}

\section{A. Principle Operation of a PLL}

The main components of a PLL are a multiplier, a low pass filter, and a VCO. The PLI block diagram is illustrated in Fig. 1.

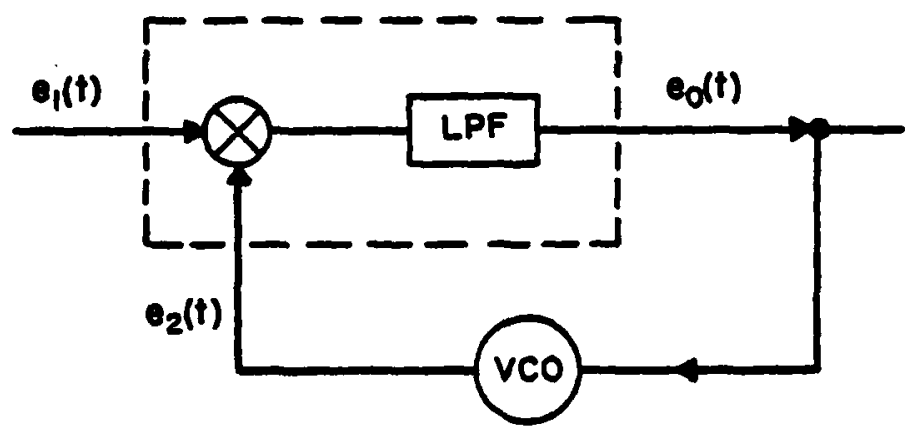

Fig. 1. Block diagram of phase locked loop.

The principle of operation of the PLL shown in Fig. 1 can be described as follows: with no input signal applied, the multipler (phase detector) output is zero and the VCO operates at its "free running frequency" $w_{2}$, With an input signal applied, the multiplier produces an error signal at its output related to the phase difference of the input signal and the VCO output signal. The error signal is low pass filtered and applied to the control terminal on the VCO, forcing the VCO frequency $w_{2}$ to approach the frequency of the input signal $w_{1}$. As suming the initial frequency difference $\left|w_{1}-w_{2}\right|$ is sufficiently small at the instant the input signal is applied, the VCO will "lock" on to the input signal, i.e., the VCO frequency will be identical to the input signal frequency with a finite phase difference. Once the loop is locked, the VCO will "track" the instantaneous phase changes of the input signal. Hence, the PLL output signal (i.e., the VCO control terminal) will vary in direct proportion to the instantaneous frequency changes of the input signal and thus successfully provide for FM demodulation and carrier tracking. The range of frequencies over which the VCO can remain in "lock" with the input signal is. called the "bold-in-range" or "lock range." The range of frequencies over which the PLL can initially acquire lock with the input signal is named the "pull-in-rangen" or "capture range."

A mathematical description of the PLI follows. Denoting the phase modulated input signal as $e_{1}(t)$ and the VCO output signal as $e_{2}(t)$ we write, 


$$
e_{1}(t)=A \sin \left(w_{1} t+\psi_{1}(t)\right)
$$

and

$$
e_{2}(t)=B \cos \left(w_{2} t+\psi_{2}(t)\right)
$$

where $\psi_{1}(t)$ and $\psi_{2}(t)$ represent the phase modulation of the input and VCO signals, respectively. If we define the phase difference between $e_{1}$ and $e_{2}$ (phase error) as $\psi(t)$, then we obtain

$$
\psi(t)=w_{1} t-w_{2} t+\psi_{1}(t)-\psi_{2}(t)
$$

and

$$
\dot{\psi}(t)=w_{1}-w_{2}+\dot{\psi}_{1}(t)-\beta e_{0}(t)
$$

where $\dot{\psi}_{2}(t)=\beta e_{0}(t)$ represents the frequency modulation of the VCO, $\beta$ denotes the VCO sensitivity, and $e_{0}(t)$ represents the input signal to the VCO.

Observation of Eq. (4) reveals that, in steady state, under locking conditions, where $\dot{\psi}(t)=0, e_{0}(t)$ is seen to be

$$
e_{0}(t)=\theta^{-1}\left[w_{1}-w_{2}+\dot{\psi}_{1}(t)\right]
$$

thus demonstrating successful FM demodulation.

To complete the loop we also recognize that $e_{0}(t)$ is the output of the low pass loop filter, i.e.,

$$
e_{0}(t)=\int^{t}\left[A B \cos \left(w_{2} \alpha+\psi_{2}(\alpha)\right) \sin \left(w_{1} \alpha+\psi_{1}(\alpha)\right)\right] h(t-\alpha) d \alpha
$$

where $h(t)$ is the impulse response of the PLL low pass filter.

B. Acoustoelectric Phase Comparator and SAW PLL Defining Equations

We now consider the special features of our system. The multiplier and low pass filter components are implemented using the nonlinear acoustic surface wave device illustrated in Fig. 2. It consists of a transformer which combines two signals $e_{1}(t)$ and $e_{2}(t)$ and whose output feeds an acoustic trans ducer. The transducer is a fingered metallic device deposited on a $Y$-cut $Z$-propagating piezoelectric $\mathrm{LiNbO}_{3}$ plate. On top of the $\mathrm{LiNbO}_{3}$ plate, a silicon plate of approximately $70 \Omega \mathrm{cm}$ resistivity is placed. The coupling of the $\mathrm{Si}$ plate is varied by changing the distance between the two plates. The action of the device is as follows. 


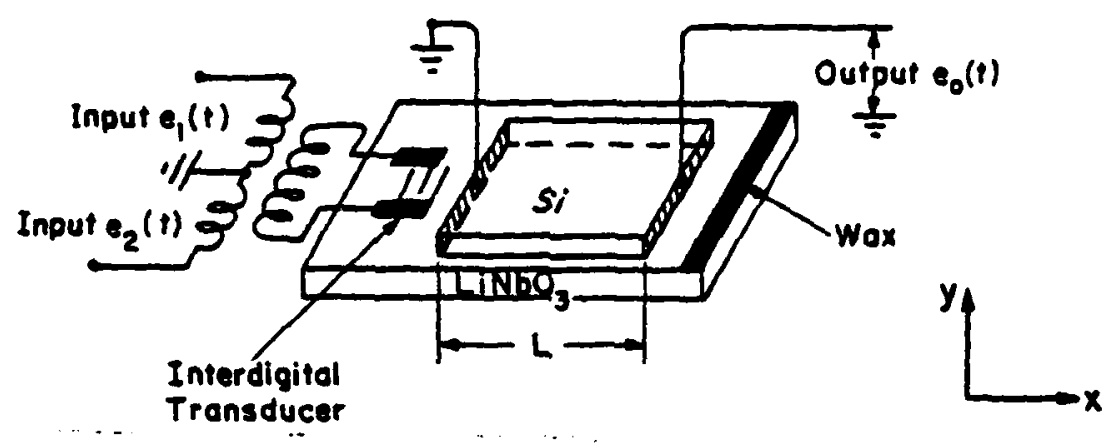

Fig. 2. Nonlinear surface acoustic wave device.

The two voltages $e_{1}(t)$ and $e_{2}(t)$ give rise to two acoustic traveling surface waves which propagate along the $\mathrm{LiNbO}_{3}$ plate in the $x$ direction. Due to the piezoelectric property of $\mathrm{LiNbO}_{3}$ two traveling wave electric fields $E_{1}(t, x)$ and $E_{2}(t, x)$ proportional to $e_{1}(t)$ and $e_{2}(t)$, respectively, are generated. The electric fields in turn generate charge carriers in the Si with a space and time variation identical to that of the electric fields. The nonlinear interactions between electric fields and charges gives rise to charge carriers and currents at sum and difference frequencies and wave numbers. The different sum frequencies charges and currents, will in turn, give rise to voltages along and across the plate.

The electric field in the semiconductor is:

$$
\begin{aligned}
E(t, y) & =E_{1}(y, w) \sin \left[\omega_{1}\left(t-\frac{x}{v}\right)+\psi_{1}\left(t-\frac{x}{v}\right)+\theta_{1}(y, w)\right] \\
& +E_{2}(y, w) \cos \left[\omega_{2}\left(t-\frac{x}{v}\right)+\psi_{2}\left(t-\frac{x}{v}\right)+\theta_{2}(y, w)\right]
\end{aligned}
$$

where $E_{1}, E_{2}, \theta_{1}, \theta_{2}$ are, respectively, the amplitude and phase of the electric fields as a function of frequency and perpendicular direction and $v$ is the velocity of the traveling acoustic wave. The charge densities in the silicon are:

$$
\begin{aligned}
\rho(y, t, x) & =\rho_{1}(y, w) \sin \left[\omega_{1}\left(t-\frac{x}{v}\right)+\psi_{1}\left(t-\frac{x}{v}\right)+\phi_{1}(w, y)\right] \\
& +\rho_{2}(y, w) \cos \left[\omega_{2}\left(t-\frac{x}{v}\right)+\psi_{2}\left(t-\frac{x}{v}\right)+\phi_{2}(w, y)\right]
\end{aligned}
$$


where $p_{1}, \rho_{2}, \phi_{1}, \phi_{2}$ are, respectively, the amplitude and phases of the induced charge densities as a function of frequency and perpendicular direction.

The coupling between these terms is due to the nonlinear current

$$
J_{N L}=i\left(p_{1} E_{1}+p_{2} E_{2}+p_{1} E_{2}+p_{2} E_{1}\right)
$$

This nonlinear current will in turn give rise to charges and electric fields at sum and difference frequencies. Specifically, if we measure the voltages between two points of the silicon plate they will contain terms at all these frequencies. If we limit ourselves only to the low frequency terms, the voltage collected in the $x$ direction over the distance $L$ and averaged over the $y$ direction will be

$$
\begin{aligned}
e_{0}(t) & =\frac{\mu}{\sigma}\left[E_{1} \rho_{1} \cos \left(\theta_{1}-\phi_{1}\right)+E_{2} \rho_{2} \cos \left(\theta_{2}-\phi_{2}\right)\right] \int_{0}^{L} u\left(t-\frac{x}{v}\right) d x+ \\
& +\frac{\mu}{\sigma} \int_{0}^{L}\left[E_{1} \rho_{2} \sin \left(\psi\left(t-\frac{x}{v}\right)+\theta_{1}-\phi_{2}\right)+E_{2} \rho_{i}\left(\sin \left(\psi\left(t-\frac{x}{v}\right)+\phi_{1}-\theta_{2}\right)\right] u\left(t-\frac{x}{v}\right) d x\right.
\end{aligned}
$$

Combining (3), (4), and (10), we obtain,

$$
\begin{aligned}
\dot{\psi}(t) & =w_{1}-w_{2}+\dot{\psi}_{1}(t)-\frac{\mu \beta}{\sigma}\left[E_{1} \rho_{1} \cos \left(\theta_{1}-\phi_{1}\right)+E_{2} \rho_{2} \cos \left(\theta_{2}-\phi_{2}\right)\right] \int_{0}^{L} u\left(t-\frac{x}{v}\right) d x- \\
& -\frac{\mu \beta}{\sigma} \int_{0}^{L}\left[E_{1} \rho_{2} \sin \left(\psi\left(t-\frac{x}{v}\right)+\theta_{1}-\phi_{2}\right)+E_{2} \rho_{1}\left(\sin \left(\psi\left(t-\frac{x}{v}\right)+\phi_{1}-\theta_{2}\right)\right] u\left(t-\frac{x}{v}\right) d x .\right.
\end{aligned}
$$

Equation (11) is the differential equation which governs the behavior of the phase locked loop. Under steady-state conditions $\psi(t)=0$ so that a constant phase shift excists which is related to the pull-in range of the PLI. The above equation has been studied experimentally and theoretically to determine the relationship between device parameters and hol-in-range and transient response of the PLL.

\section{Stability Analysis}

The transient acquisition response of the PLL is related to the stability of the system and the conditions under which locking will occur. The transient solution 
was found for the case where the parameters are almost constant with frequency. Under these conditions, it was found that the normalized equation depends only on two parameters so that Eq. (11) becomes:

$$
g_{2}(\tau)=\frac{\gamma}{\alpha}(\tau U(\tau)-(\tau-\alpha) U(\tau-a))+\int_{T-\alpha}^{T} \sin \left(W_{1} \lambda-W_{2} \lambda+\psi_{1}(\lambda)-W_{2}(\lambda)\right) d \alpha
$$

where

$$
\begin{aligned}
& \gamma=K_{1}\left[\left|\varphi_{1}\right|^{2}+\left|\varphi_{2}\right|^{2}\right] \\
& \omega_{n}=K_{2}\left|\varphi_{1} \varphi_{2}^{*}\right| \\
& \tau=\omega_{n} t \\
& W=\frac{\omega}{\omega_{n}} .
\end{aligned}
$$

and $a=\frac{k I}{W}$

Let $\dot{\psi}_{1}(T)=0$, then in steady state the VCO frequency will follow that of the input signal with a phase constant difference, i.e..

$$
\psi_{2}(\tau)=w_{1} T-w_{2} T-\psi_{s}
$$

and solving Eq.(II. 18) yields

$$
\Delta W=w_{1}-w_{2}=\gamma+a \sin \psi_{s}
$$


According to Eq. (14) the hold-in range will vary between

$Y=a<w_{1}-w_{2}<Y+a$

aree

$-1<\operatorname{cin} \psi_{\mathrm{a}}<1$

As shown in $\mathrm{Eg} .(1 \mathrm{~b})$ the mexdmum hold-in range (i.e., lock-range) for the PLL ohould be $y+a$. In order to verify dynamically the static prediction of $\mathrm{Eq}$. (14b) econputer solutions of Eq (12) were carried out for values of $Y=0, a, 2 a$ with a and $\psi_{\text {s }}$ as parameters. For a given value of a the maximum of $\psi_{s}$ for which the solution was stable were calculated and the results are plotted in Figure 3. Since $Y / a$ was kept a

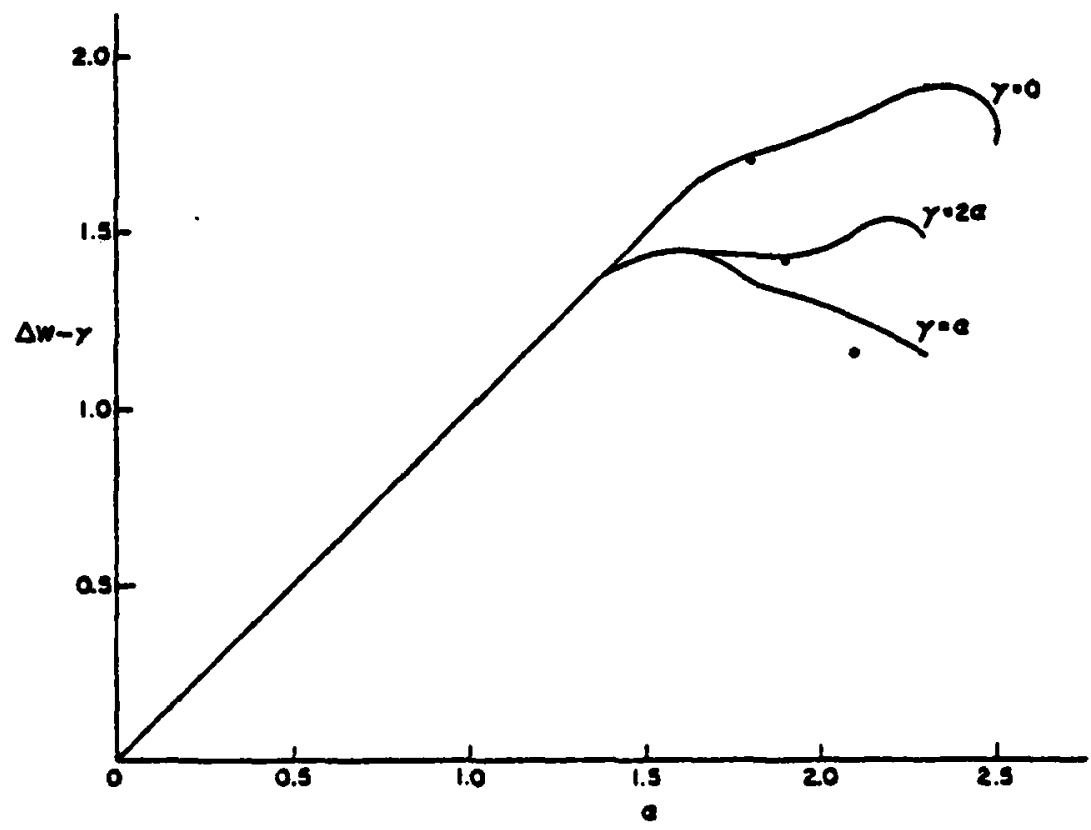

Fig. 3. PLL hold-in-range versus a.

constant for these calculations, only the vartations with a are considered.

For a small, the maximum hold-in range varies linearly with a as predicted. However, for large values of $a$, the inherent nonlinearity of the system limits the holdin range, and the value of $\Delta W$ remains a constant with an increase in the value of $a$. 
These results were verifled experimentally as shown in Figure 4 . The experimental set-up was identical to that described in References 8 and 9. Here the maximum bold-in range veraus the variations on $A / B$ (the ratio of the amplitude of the input voltage to the output of the V(O) is plotted. In this experiment the output amplitude of the VCO was kept constant and the DC component of the VCO input was compensated. Ans. lytically this corresponds to setting the fisst term on the right hand side of Eq. (12) egual to sero.

With $Y=0,5 q(14)$ reduces to

$$
\Delta \omega=\omega_{\mathbf{n}} \mathbf{a}
$$

A comparison of Figs. 3 and 4 shows that the hold-in range increases linearly but saturates for large values of $a$.

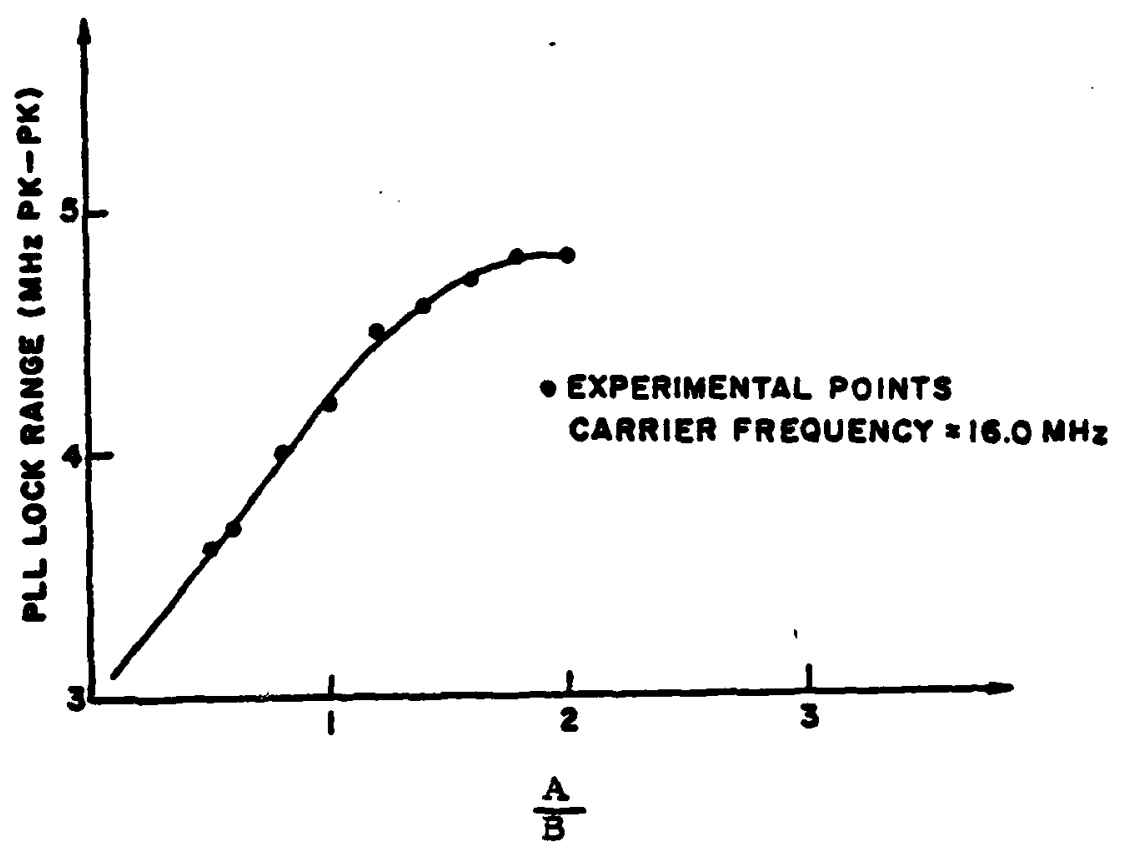

Fig. 4. PLL hold-in-range versus ratio of input eignal amplitude to VCO output igral amplitude.

In eumnary we observe that in order to get a maxdmum bold-in range and at the same time have a system with a good transient behavior we should choose an a of about 1.4 to 1.6 . 


\section{Bandwidth of PLI}

The PLL is accompanied by an IF bandpass fllter in the input and a low pass filter in the output. The closed loop bandwidth of the PLI is optimal at about twice the bandwidth of the IF flter and of course much wider than the bandwidth $2 f_{\mathrm{m}}$ of the post detection alter. 8

In order to design the accompanying fiters it is of interest to know the closed loop bandwidth of the PLI. Linearizing Eq. (12) it is easy to show that the closed loop sespouse of the PLL is given by

$$
\frac{\psi_{2}(w)}{\psi_{1}(w)}=\frac{1-e^{-j w a}}{1-w^{2}-a^{-j w a}}
$$

where $W=\omega / \omega_{n}$ is the normalized frequency. Note that the PLL is a low pass filter whose gain is one at $D C$ and whose $3 \mathrm{~dB}$ bandwidth $\mathrm{W}_{B}$ is given by the solution to the equation:

$$
4\left(1-\cos w_{B} a\right)=2\left(1-w_{B}^{2}\right)\left(1-\cos w_{B}^{a}\right)+w_{B}^{4}
$$

which for small values of a reduces to $W_{B}=a$.

A plot of $W_{B}$ rs. O is given in Figure 5. We see that the bandwidth increases with $a$ and reaches a maximum at $a=1.5$ and decreases after that. Since, as we saw earlier, an a of about 1.5 represents also a maximum bold-in range and a good transient reponse, this suggests that designing a PLL with an a between 1.4 and 1.6 would represent an optimum.

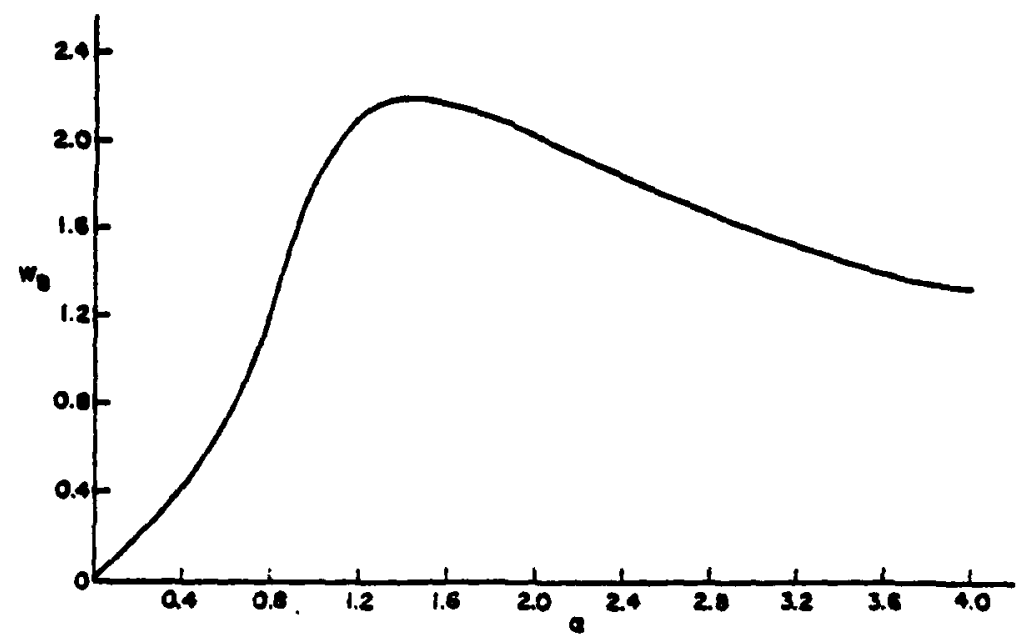

Fig. 5. PLL normalized $3 \mathrm{~dB}$ bandwidth $\left\langle W_{B}\right\rangle$ versus $a$. 
Since the circuit is nonlinear, the above results hold only in the vicinity of lock. Far from lock under transient conditions, the gain parameters will vary and the meaning of bandwidth loses its important. We showed the above phenomenon in our study of the transient conditions. Another important factor is the position of the silicon on the $\mathrm{LiNbO}_{3}$ and the distance the wave travels before reaching the silicon plate. Experimentally, we bave shown that by $a$ judicious arrangement of the silicon plate's position the effective value of a can be changed so that locking will occur without "hunting." A further study to complete the understanding of this phenomenon and improvements on the transient response and locking range of the PLL is planned.

\section{E. Noise Response}

Another process which has to be studied in analyzing a PLL is the noise response. The noise response is divided into two factors which depends on the signal to noise ratio. At high signal to noise ratio we are in the so-called above threshold situation, the input noise is assumed to be gaussian band limited by the IF filter and the noise behaves like an extra random phase on the signal input to the PLL. The problem is to find how much deterioration of the desired output occurs and this is measured by the output signal to noise ratio.

A theoretical analysis based on the above assumptions was done and it was found that because of the nonlinearity of the device the noise besides deteriorating the signal introduces also a D.C. factor which changes the locking range of the PLI shifting it to higher frequencies. The results of our theoretical and experimental studies are now described. 
When the input signal is corrupted by noise, additional a.m. and f.m. modulation appears. Let us assume that the corrupting noise is gaussian with zero expected value. Then the noise can be written as

$$
n(t)=a(t) \cos \omega_{1} t-b(t) \sin \omega_{1} t
$$

whare $a(t)$ and $b(t)$ are slowly varying gaussian stochastic processes of zero expected value. Assuming that the dominant pole of the low pass equivalent of the predetection IF fllter is "IF", then the autocorselation function of $a(t)$ and $b(t)$ is

$$
R_{a a}(T)=R_{b b}(T)=\sigma^{2} e^{-\omega} I F^{T}
$$

where $2 w_{I F}$ is the $3 \mathrm{~dB}$ bandwidth of the IF filter preceding the PLL.

With the addition of noise the input signal becomes

$$
e_{1}(t)=A \sin \left(\omega_{1} t+\psi_{1}\right)+a \cos \omega_{1} t-b \sin \omega_{1} t
$$

Rewriting we get

$$
\begin{aligned}
& e_{1}(t)=A R(t) \sin \left(\omega_{1} t+\psi_{1}+\theta(t)\right) \\
& e_{2}(t)=B \cos \left(\omega_{2} t+\psi_{2}(t)\right)
\end{aligned}
$$

where

$$
R(t)=\sqrt{1+\left(\frac{2}{A}\right)^{2}+\left(\frac{b}{A}\right)^{2}+2 \frac{a}{A} \sin \psi_{1}-2 \frac{b}{A} \cos \psi_{1}}
$$

and

$$
\tan \theta=\frac{\frac{a}{A} \cos \psi_{1}+\frac{b}{A} \sin \psi_{1}}{1+\frac{a}{A} \sin \psi_{1}-\frac{b}{A} \cos \psi_{1}}
$$

With $e_{1}(t)$ and $e_{2}(t)$ as in Eqs.(19a) and (19c)thenormalized governing relation of the PLL becomes

$$
\begin{aligned}
\dot{\psi}_{2}(T) & =\frac{A}{2 B} \int_{T=a}^{T} R^{2}(\lambda) U(\lambda) d \lambda \\
& +\frac{B}{2 \pi} \int_{T-a}^{T} U(\lambda) d \lambda+\int_{T=a}^{T} R(\lambda) \sin \left[\left(w_{1}-W_{2}\right) \lambda+\psi_{1}(\lambda)+\theta(\lambda)-\psi_{2}(\lambda)\right] U(\lambda) d \lambda
\end{aligned}
$$

whare $T$ and a were defined in Equation (13). 


\section{Rectification Effects}

We notice that the noise will contribute some rectification which will result in a shift in the bold-in range of the PLL. For small noige, assuming that in steady state the average value of the $\sin \left[\left(W_{1}-W_{2}\right) \lambda+\psi_{1}(\lambda)+\theta(\lambda)-\psi_{2}(\lambda)\right]=\sin \psi_{s}$ we get with $\psi_{1}(T)=0$ and $\dot{\psi}_{2}(T)=w_{1}-w_{2}$

$$
w_{1}-w_{2}=\frac{A}{2 B} a \overline{R^{2}}+a \frac{B}{2 A}+a \bar{R} \sin \psi_{3}=
$$

where $\overline{R^{2}}$ and $\bar{R}$ represent, respectively, the expected values of $R^{2}(T)$ and $R(T)$. Carrying out the expectations we find that

$$
\Delta w_{\text {noise }}-\Delta w_{\text {no noise }}=a \frac{\sigma^{2}}{A^{2}}\left(\frac{A}{B}+\frac{3}{2}\right)
$$

This shows that the rectification brought by noise will increase the maximum hold-in fange.

\section{Output Signal-to-Noige Radio}

We will now calculate the output signal-to-noise ratio (SNR) of the PLL under the conditions of input carrier mixed with a weak gaussian noise $\left(\frac{\sigma}{A} \ll 1\right)$. That is, we assume that the system is at equilib rium with $\psi_{2}=\left(w_{1}-w_{2}\right) \tau+\psi_{s} \cdot \psi_{1}=0$ and now we introduce noise which disturbs this equilibrium.

Since we consider weak noise, we let

$R(T)=1$

and

$$
\theta(T) \approx \frac{2}{A}
$$

Under the assumption of $\uparrow>0$ and differentiating Eq. (20) we get

$$
\bar{\Psi}_{2}(\tau)=\sin \left[\left(W_{1}-W_{2}\right) \tau+\frac{a(\tau)}{A}-\psi_{2}(\tau)\right]-\sin \left(\left(W_{1}-W_{2}\right)(\tau-a)+\frac{a(\tau-a)}{A}-\psi_{2}(\tau-a)\right)
$$

If we let $\psi_{2}(\tau)=\left(W_{1}-W_{2}\right) T+\psi_{3}+\phi_{2}(T)$ where $\phi_{2}(T)$ is the disturbance from equilibrium of $\psi_{2}(\tau)$ introduced by noise and aseume that a $\left(T / / A-\psi_{2}(T)\right.$ is small we get:

$$
\dot{\phi}_{2}(\tau)+\cos \psi_{s}\left(\phi_{2}(\tau)-\phi_{2}(\tau-a)\right)=\left\langle\frac{a(\tau)}{A}-\frac{a(\tau-a)}{A}\right) \cos \psi_{s}
$$


Lat $S_{a / A}(W)$ be the spectrum of $a / A$ and $S_{\dot{\phi}_{2}}(W)$ the spectrum of $\dot{\phi}_{2}(T)$ then

$$
s_{g_{2}}(W)=s_{2 / A}(W) \frac{2 \cos ^{2} \psi_{d}(1-\cos w a) w^{2}}{W^{4}+2 \cos \psi_{s}(\cos W a-1)\left(W^{2}-\cos \psi_{s}\right)}
$$

The spectrum of a/A is given by

$$
s_{2 / A}(W)=\frac{\sigma^{2}}{A^{2}} \frac{2 W_{I F}}{W^{2}+W_{I F}^{2}}
$$

when $W_{I F}$ is balf the bandwidth of the predetection IF filter. Assuming that the PLL is followed by a post detection fllter of bandwidth $W_{M}$ the variance of $\dot{\phi}_{2}(\tau)$ will be given by

$$
\sigma_{\phi_{2}}^{2}=\frac{\sigma^{2} 2}{A^{2} 2 \pi} \int_{0}^{W_{M}} \frac{2 W_{I F}}{W^{2}+W_{I F}^{2}} \cdot \frac{2 \cos ^{2} \psi_{s}(1-\cos W a) W^{2} d W}{W^{4}+2 \cos \psi_{s}(\cos W a-1)\left(W^{2}-\cos \psi_{s}\right)}
$$

if $W_{M} a \ll 1$, and $W_{M} / W_{I F} \ll 1$, which is usual in the case of PIL, Eq If 30) can be eanily integrated to give

$$
\sigma_{\phi_{2}}^{2}=\frac{\sigma^{2}}{\pi A^{2} W_{I F}}\left(\frac{2 W_{M}}{D^{2}} \cdot \frac{2 \arctan W_{M} D}{D^{3}}\right)
$$

where

$$
D^{2}=\frac{12-12 a^{2} \cos \psi_{s}-a^{4} \cos ^{2} \psi_{s}}{12 a^{2} \cos ^{2} \psi_{s}}
$$

Equation (25) represents the detected RMS output noise power. The detected output signal power under no noise conditions is $\left[W_{1}-W_{2}\right]^{2}$.

In a conventional first order PLL the governing equation with a weak corrupting gaueatan noise is 9

$$
\psi_{2}(t)=C \sin \left(\left(w_{1}-w_{2}\right) t+\psi_{1}(t)+\theta(t)-\psi_{2}(t)\right)
$$

where the notation used is consistent with the acoustic PLI notation previously defined. As before,

$$
\psi_{2}(t)=\left(w_{1}-w_{2}\right) t+\psi_{1}-\phi_{2}(t)
$$

where $\phi_{2}(t)$ is the disturbance due to noise.

Substituting Eg. (27) into Eq. (26) and Hnearizing we get

$$
\dot{\phi}_{2}(t)=C\left(\theta-\phi_{2}\right) \cos \psi_{\text {. }}
$$


Taking the spectrum of $\dot{\phi}_{2}(t)$ we find

$$
s_{\phi_{2}}(\omega)=\frac{s_{\theta} c^{2} \cos ^{2} \psi_{s} \omega^{2}}{\omega^{2}+c^{2} \cos ^{2} \psi_{s}}
$$

Assuming a post detection filter of bandwidth ${ }_{M}$ and $S_{\theta}$ a constant over $0<\omega<\omega_{M}$, we obtain

$$
\sigma_{\phi_{2}}^{2}=\frac{s_{\theta}}{\pi} c^{3} \cos ^{3} \psi_{s}\left[\frac{\omega_{M}}{C \cos \psi_{s}}-\arctan \frac{\omega_{M}}{C \cos \psi_{s}}\right]
$$

Comparing, we see that $C \cos \psi_{\mathrm{g}}$ in Eq. (30) corresponds to

$$
\frac{1}{D}=\sqrt{\frac{12 \alpha^{2} \cos ^{2} \psi_{s}}{12-12 \alpha^{2} \cos \psi_{s}-\alpha^{4} \cos ^{2} \psi_{s}}}
$$

is Eq. (25) which for a $\cos \psi_{s} \ll 1$ becomes

$$
\frac{1}{D}=\alpha \cos \psi_{s}
$$

This is for small a the acoustic PLL behaves as a first order PLL with closed loop bandwidth $a$ which agrees with previous interpretations. Also, substitution of Eq. (31) in Eq. (25) reveals that the r.m.s. output noise $\sigma_{\dot{\phi}_{2}}$ for the conventional first order PLL becomes identical with the acoustic $F_{L} L$.

The experimental noise study was conducted using a single tone modulated signal with a carrier frequency $f_{0}=54 \mathrm{MFz}$, a frequency deviation $\Delta f=20 \mathrm{KFHz}$, and a modulation frequency $f_{m}=1 \mathrm{KHz}$. The input carrier to noise ratio (CNR) was $10 \mathrm{~dB}$. The noise bandwidth was $40 \mathrm{KHz}$ and we used after detection a low pass filter of $35 \mathrm{kHz}$ bandwidth and a gain of 100 . We found a signal to noise ratio at the output of the low pass filter of $14 \mathrm{~dB}$. An oscillogram illustrating this noise response is shown in Fig. 10. This series of experiments will continue with the aim of establishing design rules which will maximize the detected output signal to noise ratio.

$-35-$ 


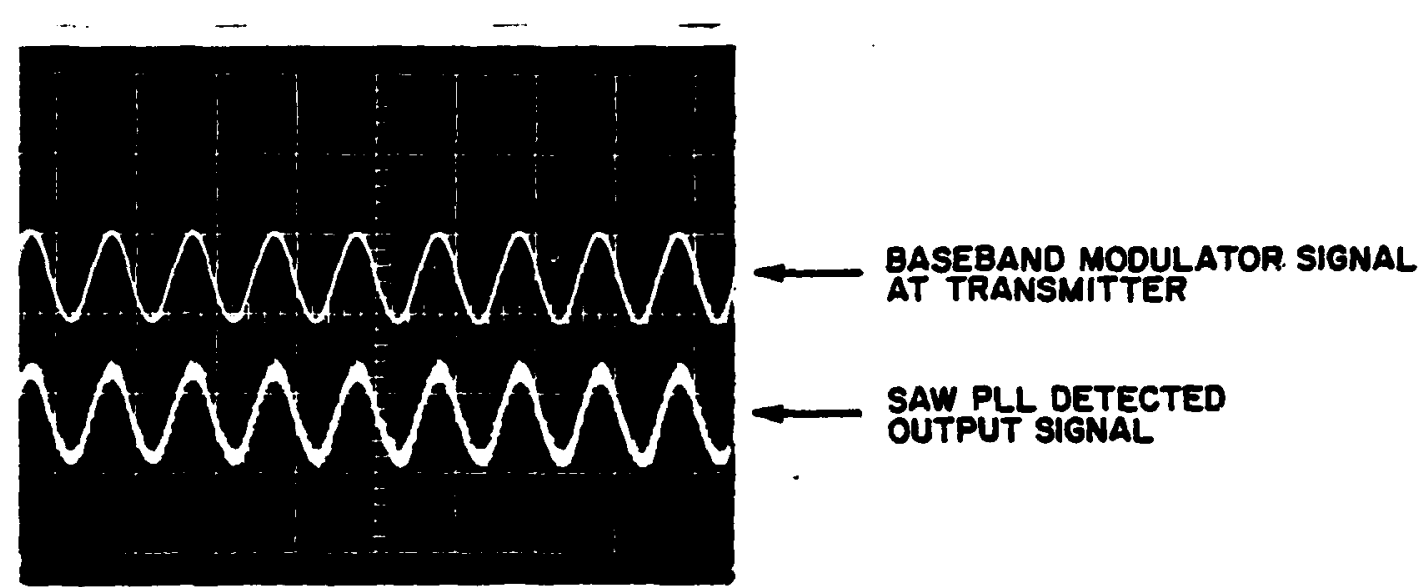

Fig. 10. Noise response of acoustoelectric PLL.

The second factor due to noise occurs at low received carrier to noise ratios below the threshold level and for cases when the phase error approaches $90^{\circ}$. In these cases, due to a rapid change in phase rate, the ouput voltage which is proportional to the instantaneous frequency of the input signal increases and decreases very rapidly giving rise to what in FM receivers is called a noise spike, i.e., "click, $n$ in the detected output signal.

There are two sources of clicks: (1) those that appear in the instantaneous frequency of the received signal due to input noise and are tracked by the demodulator and appear in the detector's output signal. These are called "clicks of the first kind." (2) Those that are generated by the demodulator itself due to "cycle slipping" events when loss of lock occurs. Such output clicks are termed "clicks of the second kind."

A theoretical study of the click rate is currently under study. Because of the bigh nonlinearity involved, it was felt that a computer aided study would be very helpful. A program was written, but because of the tremendous amount of computer time that it requires it was felt that a revision of the program was warranted which we are in the process of doing. Parallel with this endeavor, experimental studies will be pursued in the hope of optimizing the PLL noise response.

\section{F. Additional Regults}

Another important factor in our study is the extension of the frequency range of the PLL to $200 \mathrm{MHz}$. Since we do not possess a VCO capable of 
following frequency changes at this range, a hetereodyne scheme, as shown in Fig. 4, was pursued. We used a Tektronix VCO with a natural frequency of $35 \mathrm{MFz}$ and a sensitivity of $400 \mathrm{KHz} /$ volt which we mixed with an oscillator at $150 \mathrm{MFz}$ and the resulting signal at $185 \mathrm{MHz}$ was used as the input $e_{2}(t)$ to the transducer. By this method signals whose input vaiied around $185 \mathrm{MFz}$ were locked and successfully demodulated. We plan to pursue the above scheme at higher frequencies, study the PLL response at these high frequency carriers, and eventually replace the hybrid scheme with an integrated VCO.as soon as. it becomes available:

Since it was observed that the sensitivity and input FM modulation bandwidth of the VCO influences the locking range of the PIL, a mathematical as well as an experimental study of this relationship is planned. 


\section{VI.ACOUSTOELECTRIC SAW OSCILLATOR AND VOLTAGE CONTROLLED OSCILLATOR}

There are a number of ways to electronically time the frequency of a delay line type SAW oscillator, i,e., to deaign voltage controlled oscillators (VCO). Among these include: (1) vary the phase associated with the transducers and the amplifier, (2) shift the tuning condition of the transducers, and (3) vary the velocity of the surface acoustic wave. We examine and compare these technigues with the purpose of finding a configuration which will provide good FM tunability (i.e., rapid transient response and wide frequency deviation capability) and also is compatible with integrated circuit technology.

During the past year we have built an oscillator with a center frequency as high as $250 \mathrm{MHz}$ utilizing both Rayleigh and leaky type surface waves. We bave learned that it is possible to shift frequency smoothly from one mode to its adjacent mode without mode hopping, if one gradually adjusts the tming reactance associated with the interdigital transducer. However, when the tuning reactance is changed abruptly, a transition time is required for the oscillator to reach steady state, even if no mode shifting occurs. The transition time is observed to vary from $3 T_{d}$ to $14 T_{d}$, where $T_{d}$ is the delay line transit time. We have also tuned the delay-line oscillator by changing the surface conductivity of a silicon (Si) wafer which is placed in close proximity of the SAW delay line, so that the surface wave velocity is effected by the Si conductivity through electron-phonon interaction. We found that the tunability of the oscillator is less than desired due to the difficulties encountered in modulating the Si surface conductivity by a small voltage (even though in principle it can be done). Another undesired observation is due to the presence of slow states associated with the surface traps, i.e., even minutes after the controlled voltage is removed, the oscillation frequency is still influenced by the conductivity associated with the slow surface states.

A new configuration for the SAW oscillator and VCO was conceived. This new type of oscillator is derived from a successfully operated bulkwave-acoustoelectric (BW-AE) oscillator. ${ }^{10}$ The operation of the $B W-A E$ oscillator is, therefore, briefly described bere. The similarity between the proposed new SAW oscillator and the BW-AE oscillator will be discussed. Figure 1 shows the experimental configuration of the BW-AE oscillator. In Figure 1a, a CdS crystal is mounted on a piece of heat sink ( $B_{0} O$ ) to avoid excessive beating. The two opposite major faces of the CdS crystal 


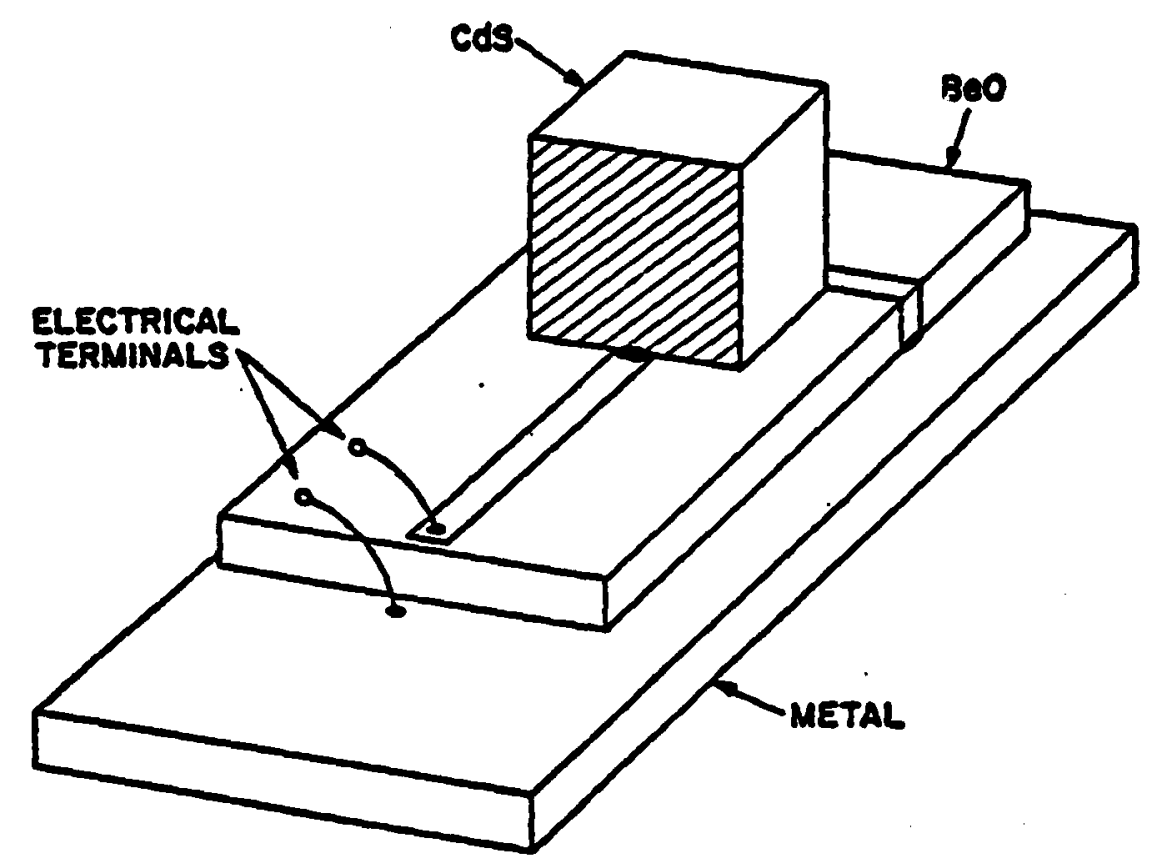

(a)

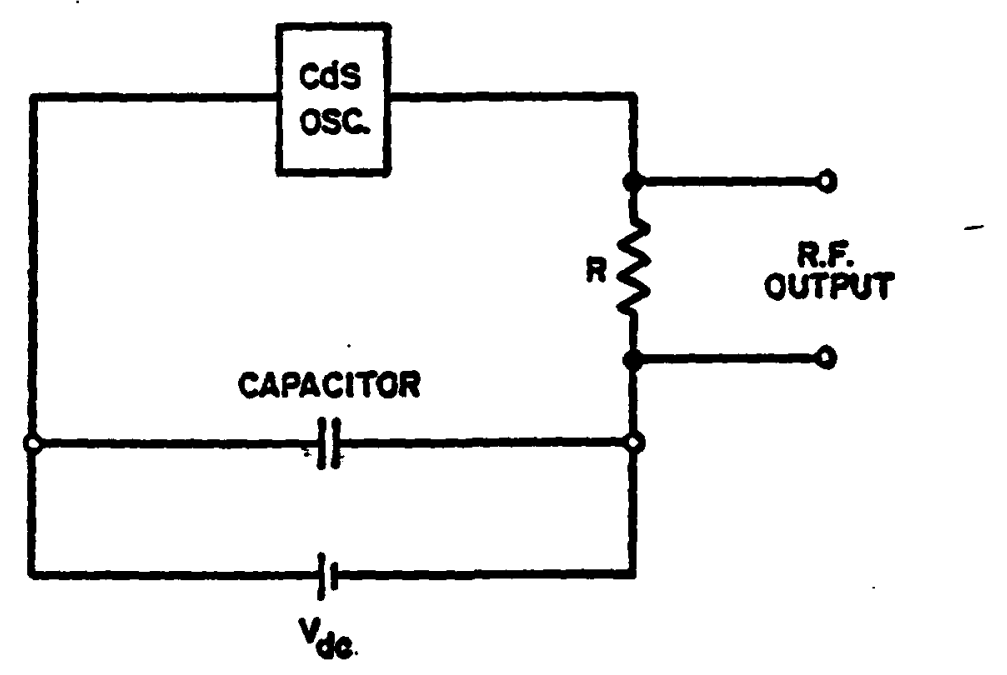

(b)

Fig. 1. (a) Device geometry of a bullowave acoustoelectric oscillator. (b) Circuit configuration of a BW-AE oscillator. 
along the hexagonal axis were polished to optical flatness and make coplanar to form a pair of parallel reflecting planes for ultrasonic waves. The polished planes were diffused with a very thin indium layer to provide electrical contacts. Since no bonds are attached to the polished faces, stress free boundary conditions are valid for sonic waves. Fig. Ib gives the circuit configuration. The output of the oscillator will be observed and taken from the viewing resistor. The physical process of the BW-AE oscillator is described as follows: when a sufficient voltage, $V_{d c}$ is applied to the CdS crystal so that the electron drift velocity exceeds the sonic velocity, the thermal phonons in a certain frequency range and in the direction of electron drift will be amplified. However, only those amplified sonic waves at frequencies which satisfy the boundary condition imposed by the parallel reflecting planes and have a net round tripgain grow into large amplitude.

Let the sonic waves that propagate in the direction of the electron flow ba referred to as forward waves and the sonic waves which propagate opposite to the electron flow as reflected waves. The net round-trip gain is defined as the amount of amplification by which the forward wave gains is greater than the loss of the reflected wave. In order for the unity gain condition required for oscillation to be satisfied in steady-state, we require

$$
\exp \left\{i\left(\frac{\omega}{v_{f}}+\frac{\omega}{v_{R}}\right) L\right\}=1
$$

where $v_{f}$ and $v_{R}$ are the velocities associated with the respective forward and reflected waves. We should point out that in the process of attaining steadystate a period of mode competition is often observed.

Fig. $2 a$ is the proposed configuration for SAW-AE oscillator. Basically, the oscillator can be operated as shown in Fig. $2 \mathrm{~b}$, which consists of a delay line, a separate-medium amplifier, and a feedback path to connect the two transducers. In case frequency modulation is desired, the modulating signal is connected in series with the applied $d-c$ voltage to modulate the velocity of the drift carriers. The basic requirement for oscillation is the same as that for the BW oscillator; the electron drift velocity should exceed the sonic velocity. In general, it requires a field intensity of several kilo-volts per 

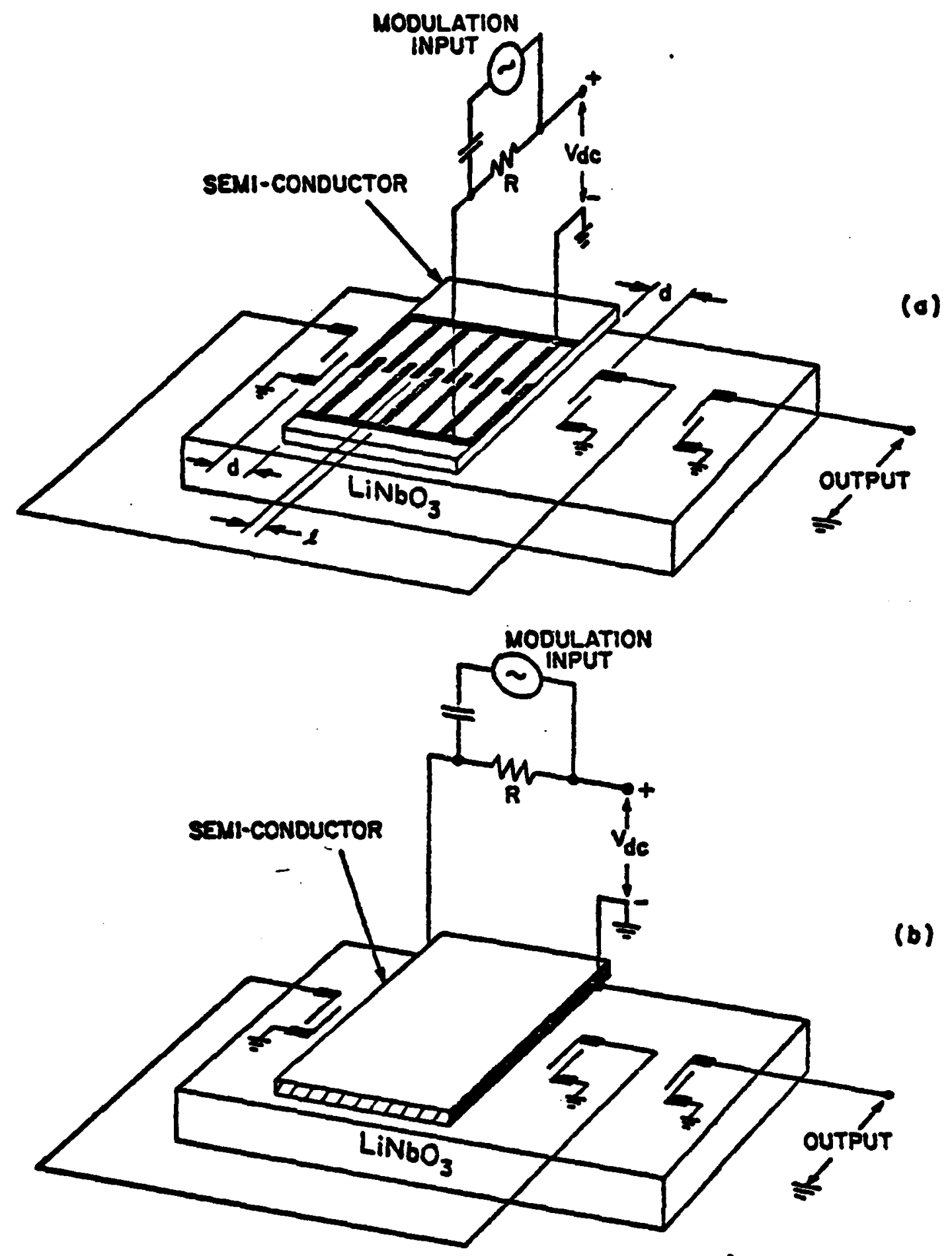

Fig. 2. (a) Proposed configuration for a SAW acoustoelectric oscillator. (b) Alternate form for a AE-SAW oscillator. 
centimeter depending on the carrier mobility of the semiconductor. For practical reasons, we propose the configuration of Fig. 2a, where a much lower voltage is required to achieve the field intensity for oscillation and for the same reason a much smaller modulating signal is needed. The configuration of Fig. $2 a$ is expected to function very much the same as that of the $B W$ oscillator. The amplified sonic wave travels alternatively in the direction of and in the direction against the electron drift. Of course, for the oscillation to occur, the sonic wave has to experience a net gain to offset the insertion loss associated with the delay line. At steady-state, the oscillation condition for Fig. $2 a$ can be written as

$$
\exp \left\{i\left(\frac{\omega}{v_{f}}+\frac{\omega}{v_{R}}\right) l+i \frac{\omega}{v_{s}}(2 d)\right\}=1
$$

where $v_{f}$ and $v_{R}$ are the SAW velocities perturbed by the conduction electrons. $v_{s}$ is the unperturbed SAW velocity. It is noted that Eq. (2) is very similar to Eq. (1). The separation between the transducer and the semiconductor $d$, should be made as small as possible.

The velocity dispersion as a function of the electron drift velocity can be depicted in Fig. 3a. The maximum change in velocity is about $\mathrm{K}^{2} / 2$, where $\mathrm{K}^{2}$ is the effective electromechanical coupling coefficient. Eq. (2) shows that the oscillation frequency depends on the SAW velocity, thus it will be a function of the applied voltage. In order to obtain larger frequency deviation, it is desirable to use a leaky surface acoustic wave, since for the leaky surface wave in the $64^{\circ}$ rotated $\mathrm{Y}$-cut $\mathrm{LiNbO}_{3}, \mathrm{~K}^{2}$ is 0.113 and for a $41^{\circ}$ rotated $\mathrm{Y}$-cut, $\mathrm{K}^{2}=0.17$, which is about six times larger than the $\mathrm{K}^{2}$ for Rayleigh wave operation. Conventional type SAW oscillator using $Y+64^{\circ}$ leaky SAW and external electronic amplifier was constructed and operated successfully at $250 \mathrm{MHz}$.

Fig. 3b shows the experimental measurement of the frequency shift of a BW-AE oscillator. The frequency shift is due to the velocity dispersion introduced by the external applied voltage. Further, we refer to Fig. $3 a$ again that the velocity dispersion is seen to occur mainly in the neighborhood where electron drift velocity equals to the sound velocity, i.e., one can modulate the frequency of the oscillator by connecting a small FM signal in series with the d.c. voltage source. This point has been demonstrated successfully in the case of BW-AE oscillator. 


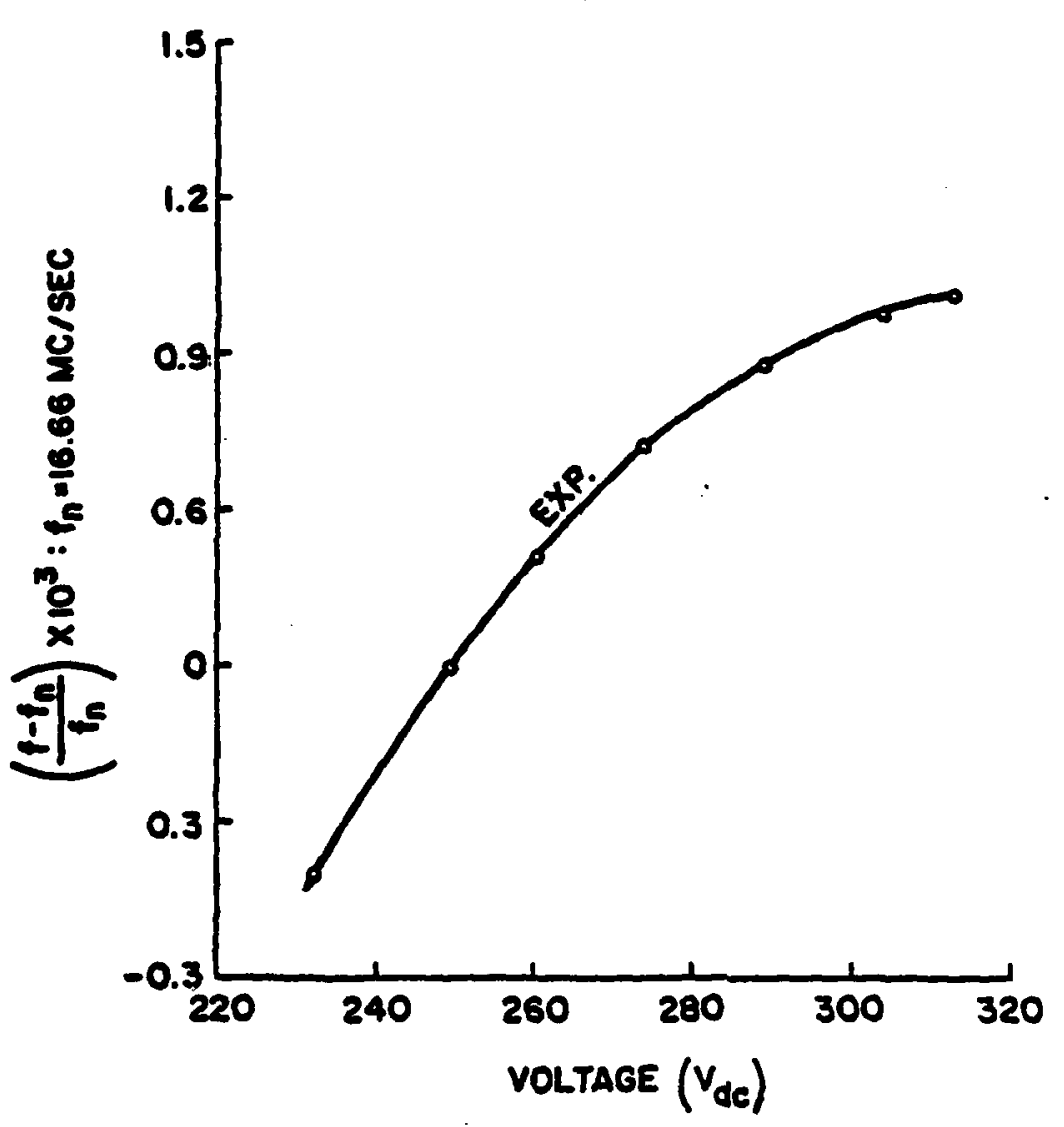

Fig. 3. (2) Velocity dispersion as a function of electron drift $v_{s}=$ unperturbed $\left(v_{d}=0\right)$ SAW velocity

$v_{d}=$ electron drift velocity

$X=$ electromechanical coupling coefficient.

(b) Normalized frequency shift of a BW-AE oscillator as a function of applied d-c voltage. 


\section{THIIN-EILM TECEINOLOGY}

Two types of thin-film configurations have been reported for the fabrication of acoustoelectric convolver, amplifier, or storage correlator. One type is to deposit ZnO film on silicon and the other is to deposit semiconductor film (InSb or CaSe) on $\mathrm{LiNbO}_{3}$. Due to difficulties associated with obtaining high quality thinfilms, the performances of those reported acoustoelectric devices have not been able to measure up to the performances of the separated medium one, thus those thin film devices have not been practical. However, due to the recent breakthrough made in ZnO thin-film deposition by T. Shiosale, S. Obnishi, et al., ${ }^{1-12}$ new enthusi asm on the monolithic, integrated acoustolectric device has reappeared on the horizon. We feel such new hopes are well founded based on the quality of $\mathrm{ZnO}$ film we have thus produced in our laboratory. (We would like to report that Dr. S. Obnishi has joined our group since the beginning of 1979. We are confident that we can develop high quality thin-film acoustoelectric devices.) Besides, we are also presently engaged in the thin-film growth of InSb. The failure of the past semiconductor film on $\mathrm{LiNbO}_{3}$ device is mainly due to the low mobility associated with such thin films. A number of factors can contribute to the case of low mobility; one is that in the process of deposition and curing, lithium or oxygen impurities from the $\mathrm{LiNbO}_{3}$ substrate migrate into the film. Our experience shows that one can obtain high mobility in Insb film with mica or glass substrate $\left(8200 \mathrm{~cm}^{2} /\right.$ volt-sec, film thickness $2700 \AA$, annealed at $509^{\circ} \mathrm{C}$ in Argon atmosphere). We believe that much higher mobility for InSb film on $\mathrm{IiNbO}_{3}$ may be obtained if one uses laser annealing, since in the process of laser annealing one can adjust the laser palse intensity such that only the thin film portion is at high temperature. The whole $\mathrm{LiNbO}_{3}$ substrate does not have to be heated-up, thus much less or no impurities are released from the substrate to the thin-film. We intend to try the laser annealing method and compare it to the conventional heat treatment. Further, since we can obtain excellent $\mathrm{ZnO}$ film (over $10 \mu \mathrm{m}$ thick on glass at present) it would be very attractive to deposit semiconductor film on $\mathrm{ZnO}$ film. The only concers bere is the velocity dispersion introduced by the $\mathrm{ZnO}$ film, since the film thicloness bere is comparable to the wavelength of the SAW. In short, we propose to fabricate and examine this structure experimentally. In the following figure we show the X-ray diffractometer pattern of a typical $\mathrm{ZnO}$ film on glass or quartz substrate. The C-axds oriented (002) films are consistently of bigh quality and clear. They are suitable for both SAW and acousto-optic applications. We also obtain comparable quality films by uaing quart substrate. In the case of quartz substrate, the substrate temperature was maintained low $\left(=200^{\circ} \mathrm{C}\right)$ during sputtering to take account of their differences in temperature coefficient. 


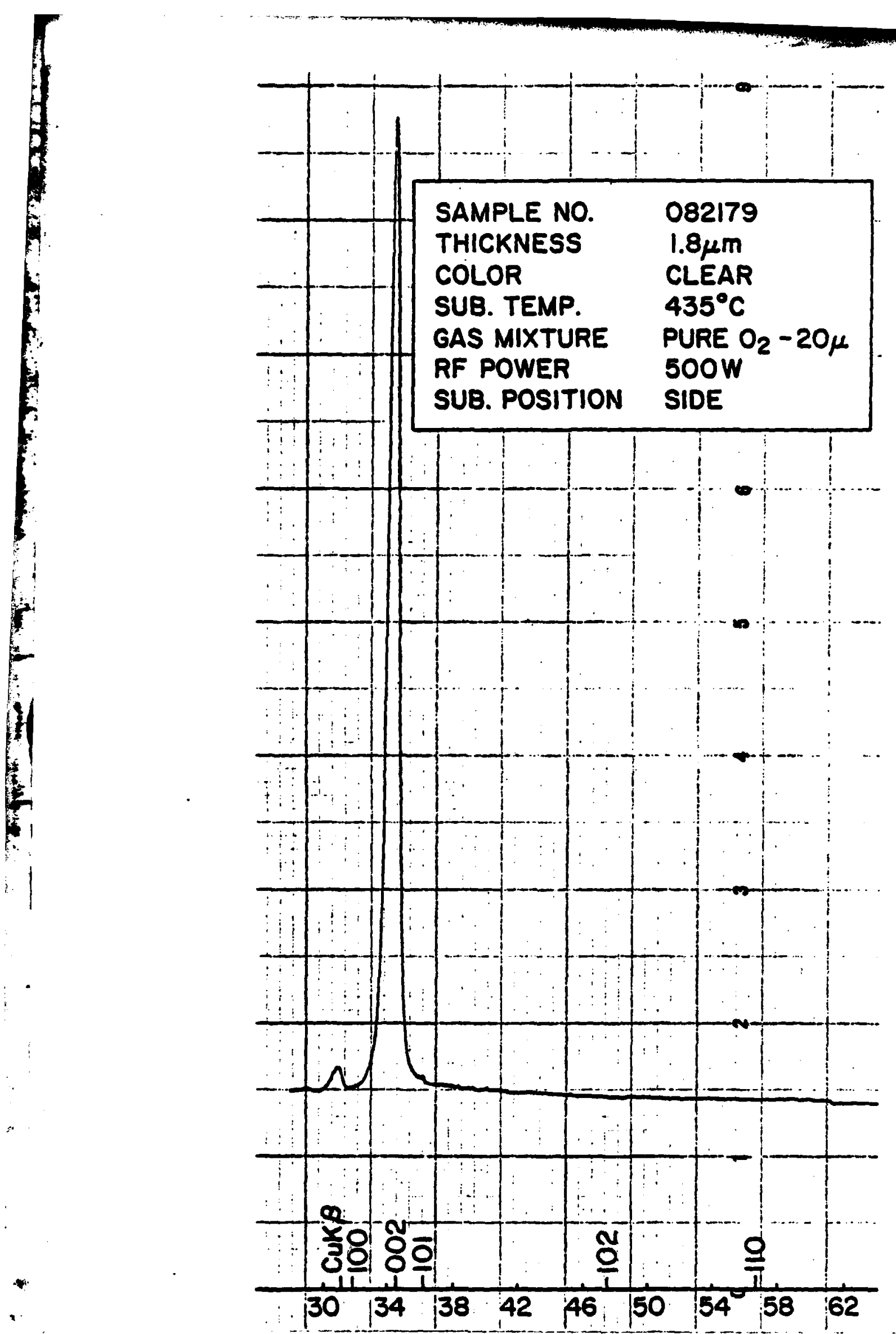

Fig. 1. X-ray diffractometer pattern of a $\mathrm{z} n \mathrm{O}$ film on glass. 


\section{REEERENCES}

1. a) K. S. Meng, H. Schachter, W-C. Wang and F. Cassara, "An Acoustic Surface Wave Phase-Locked Loop Device," International Electron Devices Meeting, p. 159, Washington, D.C., December 1974.

b) W-C. Wang, H. Schachter, F. Cassara and L. Rosenheck, "A. Novel Acoustoelectric FM Demodulator, " 1978 IEEE Ultrasonic Symposium, Cherry Hill, New Jersey.

c) W-C. Wang, H. Schachter and F. Cassara, "Acoustoelectric Surface Acoustic Wave Demodulators, " 1979 Ultrasonics Symposium Proceedings, IEEE Cat. No. 79CH1 482-9SU.

2. J. Taub and D. L. Schilling, Principles of Communication Systems, McGrawHill, Pp. 224-227 (1971).

3. H. Schachter, K. S. Meng, W-C. Wang and F. A. Cassara, An Application of Acoustic Surface Wave Devices to Communication Receivers," IEEE

Trans. on Electron Devices, Vol. 23, No. 9, September 1976, pp. 1053-1058.

4. W-C. Wang, H. Schachter and F. Cassara, "Application of Surface Acoustic Wave Devices to Communication Receivers, " Technical Report AFOSR-773353, July 31, 1978.

5. H. Schachter, F. A. Cassara and L. Rosenheck, MStability and Noise Analysis of the Acoustoelecric Phase-Locked Loop Device, " 1977 IEEE Conference Record on Acoustics, Speech, and Signal Processing, May 1977, Pp. 389

6. J.H. Cafarella, J.A. Alusow, W.M. Brown, Jr., E. Stern, "Acoustoelectric Convolvers for Programmable Matched Filtering in Spread Spectrum Systems," Proceedings of IEEE, Vol. 64, p. 756, 1976.

7. L. Rosenheck, H. Schachter, and W-C. Wang, "Acoustoelectric Real-Time Correlator, "Japanese JAP, Vol. Suppl. 18-1, p. 215, 1979.

8. M. Unleauf and R. Schulman, "Experimental Signal/Noise-Ratio Comparison of the Second Order Phase-Locked Loop and the Second Order Frequency Locked Loop, "Electronics Letters, 4, No. 26, 585-586 (Dember 27, 1968).

9. A. Viterbi, "Principles of Coherent Communication, "New York: McGrawHill, 1966).

10. D. L. White and W-C. Wang, "An Active CdS Ultrasonic Oscillator," Physical Review, 149, 628 (1966).

11. I. Shiouaki, S. Obnishi, et al., "High Rate Epitaxial Growth of ZnO Films on Sapphire by Planar Magnetron If Sputtaring System, "Journal of Crystal Growth, Vol, 45, 1978, Pp. 346-349.

12. S. Ohnishi, Y. Kirokawa, T. Shiosakd, and A. Kawabata, Japanese Journal of Applied Physics, Vol. 17, p. 773 (1971). 